\title{
生物炭的结构及其理化特性研究回顾与展望
}

\author{
张伟明修立群吴迪孙媛媛顾闻琦张鈜贵孟 军 \\ 陈温福*
}

沈阳农业大学农学院 / 辽宁省生物炭工程技术研究中心, 辽宁沈阳 110866

摘 要: 作为新兴技术, 生物炭技术及其应用在近年发展迅速, 但由于来源、材质、炭化工艺等存在较大差异, 导致 生物炭特性及应用效果千差万别, 研究结果难以比对甚至相悖, 在一定程度上阻碍了生物炭研究与应用的发展。为此, 本文从制约生物炭功效发挥的关键因素, 即生物炭的结构及理化特性入手, 系统梳理了近年有关生物炭的定义、形 成、结构、元素及其主要理化特性和调控技术等方面的研究进展, 总结分析了生物炭结构及其理化特性的共性、差 异性特征及规律, 厘清了有关生物炭特性及功能的基本观点、现状和共识。认为, 生物炭的结构及其理化特性是影响 生物炭作用、功能及效果的最主要因素, 决定了生物炭的应用领域、范围、量级、目标和方向, 采用改性或优化调控 技术是发挥生物炭功效优势、潜力与价值的关键。并从资源与环境的“循环、可持续”发展角度, 结合生物炭研究与 应用实际, 探讨了未来有关生物炭理化特性研究的基本原则和方向, 旨在为生物炭基础科学研究与应用技术发展提 供基础和参考。

关键词: 生物炭; 结构; 理化特性; 研究进展

\section{Review of biochar structure and physicochemical properties}

\author{
ZHANG Wei-Ming, XIU Li-Qun, WU Di, SUN Yuan-Yuan, GU Wen-Qi, ZHANG Hong-Gui, MENG Jun, \\ and CHEN Wen-Fu ${ }^{*}$
}

College of Agriculture, Shenyang Agricultural University / Biochar Engineering Technology Research Center of Liaoning Province, Shenyang 110866, Liaoning, China

\begin{abstract}
As a new emerging technology, biochar and its applications have been rapidly developed in recent years. However, due to large differences in carbonization materials and processes, it is difficult to compare or even contrast the results of biochar application studies, thus hindering the development of biochar applications to some extent. For this reason, our paper focuses on the key factors restricted the function of biochar, namely, the structure as well as physical and chemical properties of biochar, and then systematically presents the main research advances in recent years from the following perspectives of biochar such as definition, formation, structure, elemental composition, and other main physical-chemical properties, and property controlling-technologies. The paper analyses and summarizes the common and differential characteristics of biochar structure and physical and chemical properties and clarifies the relevant basic perspectives, statuses, trends, and consensus on the structure and properties of biochar. The structure and fundamental physical and chemical properties of biochar are believed to be the most important factors affecting the roles, function, and effects of biochar. They also determine the application field, scope, amount, objective, and direction of biochar. Therefore, the modification technology or optimal regulation technique is the key to develop the efficacy advantage,
\end{abstract}

本研究由国家重点研发计划项目“稻田生物炭基培肥产品的研制与施用技术”(2016YFD0300904-4)、“生物炭基复合肥料研制与示 范”(2017YFD0200802-02)，辽宁省高校重大科技创新平台(生物炭工程技术研究中心)项目，院士专项基金和国家水稻产业技术体系项 目(CARS01-46)资助。

This study was supported by the National Key Research and Development Program of China "the Development and Application of Biochar-based Fertilizer in Rice Soil Fertility" (2016YFD0300904-4), the State Key Special Program of Biochar-Fertilizer Technology Research and Industrialization Demonstration (2017YFD0200802-02), the Liaoning Province Major Science and Technology Platform for University (Biochar Engineering and Technical Research Center), the Special Fund for Academicians, and the National Rice Industrial Technology System (CARS01-46).

*通信作者(Corresponding author): 陈温福, E-mail: wfchen5512@126.com

第一作者联系方式: E-mail: biochar_zwm@syau.edu.cn

Received (收稿日期): 2020-03-17; Accepted (接受日期): 2020-08-19; Published online (网络出版日期): 2020-09-22.

URL: https://kns.cnki.net/kcms/detail/11.1809.S.20200922.0920.002.html 
potential and values of biochar. By further combining the research and application of biochar, the basic principles and development directions of biochar physicochemical property research in the future focusing on the physical and chemical properties of biochar are evaluated from cycle and sustainable development of resources and material perspectives. This paper aims to provide the basis and reference for the development of basic scientific science and application technology studies on biochar.

Keywords: biochar; structure; physicochemical properties; advances

生物炭(Biochar)是近年新兴的研究热点, 国内 外相关研究发展迅速。我国的生物炭研究, 自 2005 年开始迅速升温, 特别是陈温福院士于 2011 年在 《中国工程科学》上发表的“生物炭应用技术研究” 一文, 系统阐述了生物炭的性质及应用, 极大推动 了我国生物炭基础研究与应用技术的发展。时至今 日，国内外科学家在农业、环境、能源等诸多领域 开展了卓有成效的科学探索与应用研究工作, 取得 了一系列重要研究进展和成果, 可谓“百花齐放、百 家争鸣”。

然而, 在众多科学研究结果中, 我们也发现了 一些基本事实, 即不同研究结果之间差异较大且很 难进行有效的共性比对, 甚至截然相反或相悖, 对 基础研究和实践应用的指导意义和价值十分有限。 分析发现, 造成研究结果的差异性主要与原料来 源、材质, 炭化工艺条件以及应用对象、剂量、方 法等有关。此外, 由于研究、使用者的研究背景、 出发点、目标不同, 对生物炭的概念、来源、形成 及特性等缺乏系统、准确认知, 也导致其主观上对 不同生物炭研究及应用结果产生混淆或误解。因此, 十分有必要对生物炭的概念、来源、形成、结构、 特性、功能及其调控技术等进行系统梳理和总结分 析, 为生物炭研究与应用提供科学参考。

生物炭理化特性是开展生物炭研究与应用的重 要基础, 也是最为关键的一环, 决定了生物炭功效 及其应用“出口”，是生物炭发挥作用的“源泉”。目前 由于原材料来源、材质、炭化工艺及制备技术、使 用方法等方面的差异, 使得生物炭基础科学与应用 技术研究呈现“多元化、复杂化、碎片化”特征, 在不 同领域、学科均有涉及, 其研究结果或结论也有着 巨大差异性。特别是, 目前有关生物炭结构及理化 特性研究多处于“散、杂、乱”状态, 缺少系统、有效 的共性分析和比较研究。迄今, 亦鲜见针对生物炭 结构及其理化特性的系统研究和总结分析。本文在 系统梳理有关生物炭的结构及理化特性研究基础上, 分别从生物炭概念、来源、形成、结构、主要理化 特性及其调控技术等方面, 对相关主要研究进展进 行了归纳、总结和比较分析, 厘清了有关共性认知、
研究结果及结论, 并结合生物炭研究与应用实际, 从资源与环境“和谐共生、循环利用、永续发展”视 角, 对末来有关生物炭理化特性研究的基本原则、 方向及主要问题等进行了展望, 以期为生物炭基础 研究与应用发展提供基础和参考。

\section{1 生物炭的概念}

生物炭/生物质炭，作为“旧物新识”的一种“新” 事物, 追溯其产生历史, 其实早在我国两千多年前 的唐代, 就有《卖炭翁》诗云“伐薪烧炭南山中......”, 生动再现了当时的制炭场景。随着人们对制炭技术、 用途等方面的不断探索与实践，不同材质、形态的 “炭”层出不穷而又性质各异, 推动了炭化技术及其 应用研究发展, 同时也使一些研究、使用者对生物 炭概念、使用等产生了一些分歧、混淆或误解。

生物炭/生物质炭, 其译文源于英文词一一 “Biochar”, 最早用于区分生物质与化石燃料所形成 的活性炭材料 ${ }^{[1]}$ 。在 2006 年, 美国康奈尔大学 Lehman 教授丰富了“Biochar”一词的含义，将 “Biochar”定义为生物质衍生黑炭, 即一种和木炭概 念相近的材料, 可用于土壤碳库封存、改善土壤物 理与生物学特性, 以及促进植物生长的土壤改良 剂 ${ }^{[2]}$ 。在早期的生物炭相关研究中, 由于其制炭工艺 与木炭相近, 因而在国外研究中出现了“Charcoal” 和“Biochar”混用的情况, Charcoal 其含义多指现在 的“Biochar” ${ }^{33-4]}$ 。随着生物炭研究的深入, 生物炭概 念也逐渐清晰, 在 2013 年生物炭国际倡导组织 (International Biochar Initiative, IBI) 将生物炭定义 (简述)为, 生物质在限氧环境条件下通过热化学转 化获得的固体材料 ${ }^{[5]}$ 。

由于不同原料、炭化温度及工艺等条件下制备 的生物炭材料, 在结构及性质上差异很大, 使其在 应用对象、目标、范围、功效等方面存在显著差异, 导致一些分歧产生。目前, 在生物炭原料来源、炭 化条件、理化特性等方面的定性、定量界定条件还 存在不同观点, 争议来源主要为: 1)关于制炭原材料 来源, 动物体及污泥等非植物源生物质是否列入其 中；2)在炭化工艺条件方面, 高温 $\left(>700^{\circ} \mathrm{C}\right.$ 以上)快速 
裂解，是否属于常规炭化工艺方式;3)制备技术方面， 采用非传统或多途径复合、多技术融合生产的改性 生物炭或其他衍生性炭材料, 是否列为常规生物炭 的一种。上述定义、内涵、界定条件等目前尚无统 一定论，随着炭化工艺、制备技术的不断改进与创 新, 不同原理、技术及方法获得的生物炭千差万别, 也使得生物炭概念、定义等呈现“多元化、复杂化” 趋势 ${ }^{[6-8]}$ 。从目前多数研究来看, 基于生物炭来源及 应用实际，从废弃生物质资源循环利用角度，一般 认为生物炭来源于农、林等废弃生物质, 在一定炭 化温度条件下 $\left(<700^{\circ} \mathrm{C}\right)$, 在限氧或缺氧条件下热解 形成的富碳固体产物 ${ }^{[9]}$ 。

目前，尽管对生物炭概念、定义等方面的理解、 认知等还存在一些不同观点, 但随着生物炭技术的 创新与发展, 特别是多学科交叉、先进技术的不断 涌现, 生物炭结构及其理化特性研究将不断推向深 入, 届时生物炭概念、定义等将得到逐步统一、完 善和发展。

\section{2 生物炭的形成过程}

生物炭, 是由生物质经热裂解反应过程后而形成 的一种产物 ${ }^{[10-12]}$ 。在热裂解过程中, 生物质会通过分 子内、分子间的重排作用反应而形成由芳香多环等结 构形成生物炭, 以及生物油、混合气等物质 ${ }^{[13-14]}$ 。

不同材质生物质的炭化形成过程存在差异。一 般情况下, 制炭原料可分为木质和非木质纤维素类 生物质 ${ }^{[15-16]}$ 。其中, 木质纤维素类生物质主要来源 于植物类废弃物 ${ }^{[17-18]}$, 其主要成分为纤维素、半纤 维素、木质素等 ${ }^{[19-21]}$ 。而非木质纤维素类生物质则 主要包括动物粪便、一部分植物及其衍生物等 ${ }^{[16]}$, 其主要成分为蛋白质、脂类、糖类、无机物以及部 分木质素、纤维素 ${ }^{[22]}$ 。一般情况下，木质纤维素类 生物质在 $200 \sim 260^{\circ} \mathrm{C}$ 时开始发生热裂解反应，半纤 维素的分支聚合物和短侧链先分解为低聚糖, 其后

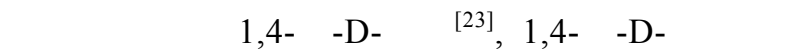
过脱水、脱羧、芳构化及分子内缩合等过程后形成 炭, 或分解形成低分子量的生物油及混合气 ${ }^{[24]}$ 。在 $300^{\circ} \mathrm{C}$ 左右纤维素开始分解, 先解聚成低聚糖, 随后 糖苷键断裂生成 D-吡喃葡萄糖, 在分子内重排形成 左旋葡萄糖聚糖 ${ }^{[25]}$, 其中一部分左旋葡聚糖经过脱 羧、芳构化、分子内缩合等过程后形成炭，另一部 分在经重排、脱水过程后形成羟甲基糠醛, 进一步 形成生物油及混合气 ${ }^{[24]}$ 。在 $400^{\circ} \mathrm{C}$ 时木质素开始大
量分解为自由基，并通过自由基取代、加成、碳碳 偶合等过程后形成生物炭、生物油和混合气 ${ }^{[26-27]}$ 。 在超过 $500^{\circ} \mathrm{C}$ 时, 生物炭孔隙内的聚合物、挥发性化 合物会发生再聚合反应, 形成次生炭或经过重组产 生分子量较高的焦油 ${ }^{[28-30]}$ 。而非木质纤维素类生物 质, 在 $200^{\circ} \mathrm{C}$ 左右开始发生热解反应，蛋白质、脂类、 碳水化合物等有机物的低键能键(如氢键、氢氧化钙 键)发生断裂 ${ }^{[16]}$, 键断裂随温度升高而增强, 主要热 解过程发生在 $300 \sim 600^{\circ} \mathrm{C}$, 最终热解形成生物炭、生 物油及混合气 ${ }^{[16,31]}$ 。一般情况下, 由于非木质纤维素 类生物质中的蛋白质、脂类以及核酸中含有大量氮、 磷和氧, 使其热解行为更为复杂而多变 ${ }^{[32]}$ 。

在生物质热解炭化过程中，完成了由“生物质一 炭”的物质形态转变, 形成了极其丰富的多微孔碳架 结构，以及不同种类的表面官能团、有机小分子及 矿物盐等 ${ }^{[33]}$, 为其作为吸附、载体等功能材料奠定 了重要基础, 使其可在农业、环境、能源等领域广 泛应用 ${ }^{[34]}$ 。

\section{3 生物炭的结构}

\section{1 表面及内部结构表征}

生物炭的结构, 主要由生物质原有结构在经过 失水、活性物质挥发、断裂、崩塌等一系列热解炭 化过程后重构形成 ${ }^{[35]}$ ，其“骨架”结构由稳定的芳香 族化合物和矿物组成 ${ }^{[4,36]}$ ，孔隙结构则由芳香族化 合物和其他功能基团组成 ${ }^{[37-38]}$ 。生物炭的孔隙表征, 参照国际纯粹与应用化学联合会(International Union of Pure and Applied Chemistry, IUPAC)的活性炭孔隙 分类可分为微孔 $(<2 \mathrm{~nm})$ 、中孔(2 50 nm)、大孔(> $50 \mathrm{~nm})$, 生物炭中的孔隙多以微孔为主 ${ }^{[39]}$ 。生物炭的 结构表征与其炭化温度条件有关, 随着炭化温度升 高, 生物炭的非晶态碳结构逐步转化为石墨微晶态 结构, 晶体尺寸扩大、结构更加有序 ${ }^{[40]}$ (图 1)。

\section{2 生物炭结构形成的主要影响因素}

制炭原料与炭化温度，是生物炭结构形成的主 要影响因素 ${ }^{[41]}$ 。不同生物质原料在结构、内含物等 方面存在本质差异，导致其在炭化后的结晶度、交 联和分支等结构特征上差异显著 ${ }^{[42-43]}$ 。木质素含量 高的生物质(如竹子、椰子壳等)在炭化后的大孔结构 增多, 而纤维素含量高的生物质(如植物外壳)在炭 化后形成的结构多以微孔为主 ${ }^{[44]}$, 这也使纤维素类 生物炭的表面积(112 642 $\left.\mathrm{m}^{2} \mathrm{~g}^{-1}\right)$, 一般高于非纤维 素类生物炭(3.32 94.2 $\left.\mathrm{m}^{2} \mathrm{~g}^{-1}\right)^{[45-47]}$ 。 


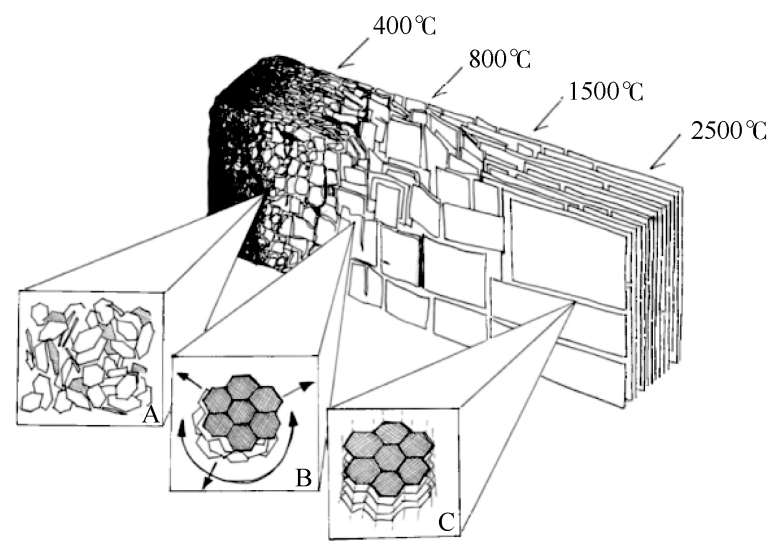

图 1 不同炭化温度下的生物炭结构表征示意图 ${ }^{[4]}$

Fig. 1 Biochar structure representation under different carbonization temperature ${ }^{[4]}$

$\mathrm{A}$ : 生物炭结构中芳香族碳增加, 主要以无定型碳为主。B: 生物 炭结构中涡轮层状芳香碳增加。C: 生物炭结构趋于石墨化。

A: the aromatic carbon in biochar structure is increased, mainly part is amorphous carbon. B: the sheets of turbostratic aromatic carbon in biochar structure is increased. C: the biochar structure becomes graphitic.

炭化温度条件, 也是影响生物炭结构的重要因 素。在热解炭化过程中, 随着炭化温度升高, 生物炭 中的挥发性物质逐渐热解分离、挥发，形成更多新 孔隙和不规则、粗糙的炭粒蚀刻表面 ${ }^{[48-49]}$ 。当炭化 温度高于 $700^{\circ} \mathrm{C}$ 时，生物炭表面微孔结构开始出现 破坏，超过 $800^{\circ} \mathrm{C}$ 时生物炭的多孔碳架结构表现不稳 定, 坍塌现象发生 ${ }^{[50-51]}$ 。炭化停留时间, 也可在一定程 度上影响生物炭的结构。当炭化温度为 $500 \sim 700^{\circ} \mathrm{C}$ 、 停留时间在 $2 \mathrm{~h}$ 以内时，生物炭的孔隙度随停留时间 延长而增大，但当超过 $2 \mathrm{~h}$ 后则表现为负效应 ${ }^{[52]}$ 。一 般情况下，慢速热解炭化更有利于生物炭的多微孔 形成 ${ }^{[23]}$, 而在快速热解炭化过程中, 由于未完全热 解的类焦油等物质可能会滞留、堵塞生物炭孔隙, 不利于生物炭的多微孔结构形成 ${ }^{[53]}$ 。

生物炭的多微孔结构是其发挥作用的重要基 础, 不同原料、炭化工艺条件下制备的生物炭结构 表征、特性差异明显，实际应用中可根据不同场景、 目的及应用目标的需求, 通过材质定向篮选、炭化 工艺定向调控等措施获得具有一定预期结构特征 的生物炭，从而充分发挥生物炭“构一效”优势及其 作用潜力。

\section{4 生物炭的主要理化特性}

\section{1 酸碱性}

生物炭一般呈碱性, 但也有酸性表现, 其 $\mathrm{pH}$ 变 幅范围在3 13 $3^{[54-55]}$ 。一般情况下, 原料中的灰分含量
越多, 其制备的生物炭 $\mathrm{pH}$ 越高 ${ }^{[56]}$ 。在材质上, 由于 非纤维素类生物质制备的生物炭具有较高灰分含量, 因此，一般非纤维素类生物炭 $\mathrm{pH}$ 高于纤维素类生物 炭 ${ }^{[57-58]}$ 。而在相同炭化工艺条件下, 不同材质生物炭 的 $\mathrm{pH}$ 表现为禽畜粪便 $>$ 草本植物 $>$ 木本植物 ${ }^{[56,59]}$ 。

一般认为, 生物炭 $\mathrm{pH}$ 随炭化温度升高而提 高 ${ }^{[55]}$ 。在较高炭化温度条件下, 会加速酸性官能团 的分解如- $\mathrm{COOH}$ 、- $\mathrm{OH}$ 等, 使生物炭 $\mathrm{pH}$ 提高 ${ }^{[46,60]}$ 。 研究发现, 当温度由 $200^{\circ} \mathrm{C}$ 升高到 $800^{\circ} \mathrm{C}$ 时, 生物炭

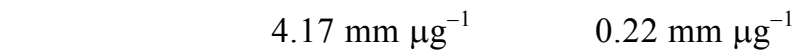
碱性官能团由 $0.15 \mathrm{~mm} \mathrm{\mu g}^{-1}$ 升高到 $3.55 \mathrm{~mm} \mathrm{\mu g}^{-1}$, 而 $\mathrm{pH}$ 则由 7.37 提高至 $12.40^{[61]}$ 。较高炭化温度也有利 于促进灰分中碱金属 $(\mathrm{Na} 、 \mathrm{~K})$ 或碱土金属 $(\mathrm{Ca} 、 \mathrm{Mg})$ 等离子化合物的形成，如 $\mathrm{KOH} 、 \mathrm{NaOH} 、 \mathrm{MgCO}_{3}$ 、 $\mathrm{CaCO}_{3}$ 等, 从而提高生物炭 $\mathrm{pH}^{[60]}$ 。此外, 炭化停留 时间亦可在一定程度上影响生物炭 $\mathrm{pH}$, 表现为炭化 停留时间越长而 $\mathrm{pH}$ 越高 ${ }^{[62-63]}$, 但加热升温速率对 生物炭 $\mathrm{pH}$ 的影响不大 ${ }^{[64]}$ 。

生物炭的酸碱性, 是影响其特性及功能的重要 指标之一。从目前研究来看, 由于原料、炭化工艺 及制备技术等不同, 生物炭 $\mathrm{pH}$ 变化范围较广, 基本 覆盖了“酸性、中性、碱性”不同状态。在实际生产 和应用过程中, 可通过炭化工艺调控、改性等技术 方法获得一定酸碱范围的生物炭材料, 根据使用目 的、目标科学施用。

\section{2 比表面积}

生物炭丰富的多微孔结构, 使其具有较大的比 表面积 ${ }^{[65-67]}$ 。生物炭的比表面积, 与其制炭原料、 炭化温度等有关。一般情况下，纤维素类生物质因 其具有丰富的内部孔隙, 炭化后形成的生物炭会保 留原有生物质细微孔隙结构, 比表面积大幅提高, 而非纤维素类生物质的孔隙结构相对较少, 因此炭 化后的生物炭比表面积小于纤维素类生物炭 ${ }^{[68]}$ 。

炭化温度, 是影响生物炭比表面积的重要因素 之一。当炭化温度在 $400^{\circ} \mathrm{C}$ 以下时, 生物质在炭化过 程中形成的孔隙率降低, 可能与生物质中挥发性物 质的热解分离和挥发不完全等有关 ${ }^{[69-70]}$ 。随着炭化 温度升高, 生物质分离、释放出更多挥发性物质, 孔 隙更多、比表面积增大 ${ }^{[24,71-73]}$ 。但是, 在高于 $800^{\circ} \mathrm{C}$ 时, 生物炭中的多孔结构会发生部分坍塌, 从而堵

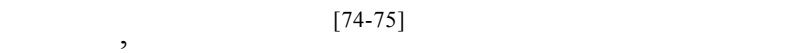
炭的比表面积, 一般情况下随着炭化温度升高, 生 物炭的灰分含量增加, 会堵塞部分生物炭孔隙, 从 
而使其比表面积降低 ${ }^{[76]}$ 。

比表面积大, 是生物炭作为载体和吸附功能材 料的重要基础特性之一。在生物炭制备过程中, 比 表面积大小可通过改性、调控工艺流程等进行一定 程度的定向调控。

\section{3 元素组成}

生物炭主要由 $\mathrm{C} 、 \mathrm{O} 、 \mathrm{H} 、 \mathrm{~N} 、 \mathrm{~K} 、 \mathrm{Ca} 、 \mathrm{Na} 、 \mathrm{Mg}$ 等元素组成 ${ }^{[66,77]}$ 。其中, $C$ 元素主要为芳香环形式的 固定碳，而碱金属 $(\mathrm{K} 、 \mathrm{Na}$ 等 $)$ 、碱土金属 $(\mathrm{Ca} 、 \mathrm{Mg})$ 等则以碳酸盐、磷酸盐或氧化物形式存在于灰分 中 ${ }^{[60,78]}$ 。生物炭中的元素种类、含量, 主要与原材 料有关 ${ }^{[79-80]}$ 。研究认为, 纤维素类生物炭中的 $\mathrm{C}$ 含 量高于非纤维素类生物炭, 木材、竹类生物炭的 $\mathrm{C}$ 含量较高 ${ }^{[60,81]}$ 。而在其他元素中, 纤维素类生物炭中 的 $\mathrm{K}$ 含量相对较高 ${ }^{[77,82-84]}$, 非纤维素类生物炭中的 $\mathrm{Ca} 、 \mathrm{Mg} 、 \mathrm{~N} 、 \mathrm{P}$ 等元素高于纤维素类生物炭 ${ }^{[85]}$ 。炭 化温度, 也是影响生物炭元素含量及组成的重要因 素。一般情况下，生物炭中的 C、碱金属及其矿化物 含量随炭化温度升高而提高, 而 $\mathrm{N} 、 \mathrm{H} 、 \mathrm{O}$ 等元素含 量则随炭化温度升高而降低 ${ }^{[77,86-88]}$ 。

\subsection{1 元素形态及有效性生物炭中的 $\mathrm{C}$, 主要} 为芳香族碳, 以芳香环、不规则叠层堆积存在, 使生 物炭具有稳定性高、抗分解能力强特性 ${ }^{[4,89-90]}$ 。生物 炭还含有一定有机碳, 主要为未完全碳化的生物质 和脂肪酸、醇类、酚类、酯类化合物, 以及类黄腐 酸、胡敏酸等物质组分, 一般在新制备生物炭、低 温热解炭化形成的生物炭以及非纤维素类生物炭中 的含量相对较高 ${ }^{[54,62-91]}$ 。生物炭中的 N、P、K 及其 他无机盐离子等元素, 可作为植物、微生物等生命

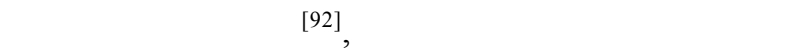
量、有效性等存在差异，一般富含养分原料制备的 生物炭, 其含有的营养元素相对较高, 反之亦然 ${ }^{[93]}$ 。

生物炭中的元素有效性, 与生物炭的新鲜程 度、 $\mathrm{pH}$ 、炭化温度等条件有关 ${ }^{[94]}$ 。一般情况下，新 制备的生物炭可释放更多 N、P、K 等养分元素, 而 陈化的生物炭在养分释放量、速度等方面相对弱一 些 ${ }^{[95-97]}$ 。生物炭 $\mathrm{pH}$ 也会影响其元素有效性, 当 $\mathrm{pH}$ 在 2 7 之间时, 生物炭释放 $\mathrm{PO}_{4}{ }^{3-}$ 和 $\mathrm{NH}_{4}{ }^{+}$随 $\mathrm{pH}$ 提高 而降低, 而 $\mathrm{K}^{+}$含量则保持相对稳定 ${ }^{[53]}$, 而当 $\mathrm{pH}$ 从 8.9 降至 4.5 时, 生物炭中的 $\mathrm{Ca} 、 \mathrm{Mg}$ 元素释放量会 增加 ${ }^{[98]}$ 。此外, 炭化温度亦会影响生物炭中的元素 状态，随着炭化温度提高，生物炭中的碱金属等矿 物组分趋于结晶态, 使其水溶性降低 ${ }^{[78,99-100]}$ 。当热
解温度由 $300^{\circ} \mathrm{C}$ 升至 $600^{\circ} \mathrm{C}$ 时, 生物炭中的可溶性 $\mathrm{PO}_{4}{ }^{3-}$ 由 $430 \mathrm{mg} \mathrm{kg}^{-1}$ 下降到 $70 \mathrm{mg} \mathrm{kg}^{-1[101]}$ 。

4.3.2 潜在有害元素生物炭中的元素, 绝大部 分对土壤、植物及环境等有益或无害, 但受制炭原 料的材质、内含物及其炭化过程等因素影响, 某些 情况下还可能含有一定潜在有害元素或物质。研究 表明, 生物炭中的潜在有害物质主要包括有机污染 物和重金属两大类 ${ }^{[102]}$ 。

第一类: 有机污染物。生物炭中的有机污染物 主要包括多环芳烃(polycyclic aromatic hydrocarbons, PAHs)、多氯二苯并二噁英(polychlorinated dibenzo dioxins，PCDDs)、多氮二苯并呋喃(polychlorinated dibenzo furans, PCDFs), 以及一些挥发性有机化合 物、二甲苯酚、甲酚、丙烯醛、甲醛等 ${ }^{[102]}$ ，这些物 质主要在热解炭化过程中产生, 过量可能对土壤、 微生物、植物等健康构成风险 ${ }^{[103-104]}$ 。研究发现, 生 物炭中的多环芳烃主要与原料中的木质素、纤维素 和半纤维素含量有关 ${ }^{[105]}$ ，一般情况下木质素含量高 的原材料在炭化后的多环芳烃含量较低, 如木质生 物炭的多环芳烃低于秸秆类生物炭 ${ }^{[106]}$ 。不同种类生 物炭的有机污染物含量差异显著, 多环芳烃(PAHs) 含量从 $<0.1 \mathrm{mg} \mathrm{kg}^{-1}$ 到> $10,000 \mathrm{mg} \mathrm{kg}^{-1}$ 表现不等 ${ }^{[107]}$, 生物炭国际倡导组织(IBI)建议生物炭的多环芳烃含 量应控制在 6 20 mg kg-15]。不同炭化温度，也会影 响生物炭的潜在污染物含量及形态。一般情况下, 低温制备的生物炭含有较高浓度的低分子量多环芳 烃，而在高温下制备的生物炭，其高分子量多环芳 烃的浓度相对较高 ${ }^{[108]}$ 。炭化过程中, 可通过输入 $\mathrm{O}_{2}$ 和 $\mathrm{CO}_{2}$ 作为载气来降低 $\mathrm{PAH}$ 含量 ${ }^{[107]}$, 而在炭化后 可通过在厌氧环境中冷却生物炭亦有助于降低其多 环芳烃含量 ${ }^{[107]}$ 。

第二类: 重金属。生物炭中的重金属元素, 主要 包括 $\mathrm{Cd} 、 \mathrm{Cu} 、 \mathrm{Cr} 、 \mathrm{Ni} 、 \mathrm{~Pb} 、 \mathrm{Zn} 、 \mathrm{Hg}$ 等 ${ }^{[102]}$ ，其含量 与原材料密切相关，一般认为非纤维素类材质制备 的生物炭，其重金属含量高于纤维类生物炭 ${ }^{[16]}$ 。在 炭化过程中, 生物炭中残留的重金属形态相对稳定, 但其直接毒性、生物有效性比炭化前降低 ${ }^{[16]}$, 炭化

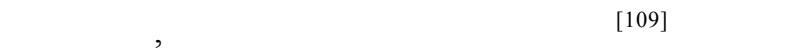
性越低 ${ }^{[109-110]}$ 。生物炭国际倡导组织(IBI)经过风险评 估后，对生物炭中的有害元素含量上限提出了明确 建议(表 1) ${ }^{[111]}$ 。

生物炭中的潜在有害元素含量及其有效性，若 在安全范围内对土壤、作物及环境的影响很小，但 
表 1 生物炭中有害元素含量上限阈值 ${ }^{[111]}$

Table 1 Maximum allowed thresholds of the toxic elements in biochar $^{[111]}$

\begin{tabular}{lc}
\hline $\begin{array}{c}\text { 有害元素 } \\
\text { Toxic element }\end{array}$ & $\begin{array}{c}\text { 含量 } \\
\text { Content }\left(\mathrm{mg} \mathrm{kg}^{-1}\right)\end{array}$ \\
\hline 砷 Arsenic & $13-100$ \\
镉 Cadmium & $1.4-39.0$ \\
铜 Copper & $143-6000$ \\
铬 Chromium & $93-1200$ \\
镍 Nickel & $47-420$ \\
铅 Lead & $121-300$ \\
锌 Zinc & $416-7400$ \\
承 Mercury & $1-17$ \\
钼 Molybdenum & $5-75$ \\
钴 Cobalt & $34-100$ \\
\hline
\end{tabular}

在超限投入条件下可能对生态环境安全造成一定风 险。因此, 在规模化、工程化实施过程中, 要严格篮 选、控制原料来源, 或采用如在炭化前添加磷酸二 氢钙或沸石来降低原材料中重金属的生物利用度 ${ }^{[112]}$ 等一些预处理措施来降低其重金属含量, 最大限度 降低生物炭中的潜在有害元素风险，使其在安全、 可控范围内。同时, 通过调控炭化工艺条件及参数, 尽可能减少生物炭中的污染物及有害元素残留, 降 低其生态环境安全风险。

生物炭的元素组成、含量及形态, 是生物炭的 重要特性及其功效发挥的“源泉”。主要体现在: 1)释 放 $\mathrm{N} 、 \mathrm{P} 、 \mathrm{~K} 、 \mathrm{Ca} 、 \mathrm{Mg}$ 等大量及中微量元素, 为土 壤提供一定外源养分, 促进植物生长 ${ }^{[113]} ; 2$ )生物炭 的重要组分一一灰分, 除含有一定碱金属物质外, 可在吸附金属元素等过程中发挥关键作用 ${ }^{[114]}$ 。

\section{4 表面化学性质}

\subsection{1 表面官能团生物质中的纤维素、半纤维}

素、蛋白质、脂肪等, 经热解炭化后在生物炭表面 及内部形成大量羧基、羰基、内脂基及羟基、酮基 等多种类型官能团，其中大多为含氧官能团或碱性 官能团，使生物炭具有良好的吸附、亲水/疏水，以 及缓冲酸碱、促进离子交换等特性 ${ }^{[38,115-116]}$ 。

生物炭的表面官能团与制炭原料、炭化温度条 件密切相关 ${ }^{[46]}$ 。不同原材料中, 非纤维素类生物炭 比纤维素类生物炭含有更多的 N、S 官能团 ${ }^{[117]}$ 。在 不同炭化温度条件下, 生物炭官能团的数量、密度 随炭化温度升高而下降 ${ }^{[92]}$ 。在 $185 \sim 200^{\circ} \mathrm{C}$ 时, 生物炭 表面官能团种类不会发生明显变化 ${ }^{[46,118]}$, 而当温度 达到 $300^{\circ} \mathrm{C}$ 时, 羧基、羰基含量则快速上升至最高点,
此后随炭化温度升高而降低 ${ }^{[72]}$ 。至 $400 \sim 550^{\circ} \mathrm{C}$ 时, 生 物炭的脂肪族官能团随温度升高而逐渐消失 ${ }^{[70,118]}$, 当温度达到 $600^{\circ} \mathrm{C}$ 时, 烷基碳官能团消失 ${ }^{[46]}$ 。

\subsection{2 阳离子交换量阳离子交换量(cation ex-} change capacity, CEC), 是决定生物炭表面化学特性 的基础指标，也是衡量其离子交换、吸附性能的重

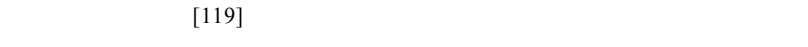
料、炭化温度等有关 ${ }^{[120]}$ 。研究发现, 非纤维素类生 物炭的 CEC 比纤维素类生物炭高, 而原料发酵后制 备的生物炭 CEC 要高于未发酵原料制备的生物

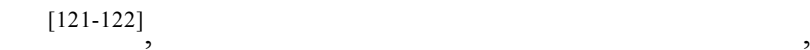
导致其阳离子交换量存在差异 ${ }^{[66]}$ 。在不同炭化温度 条件下, 较低温度制备的生物炭表面含有更多含氧 官能团, CEC 较高, 而在较高温度下制备的生物炭, 其含氧官能团被破坏，表面负电荷减少，CEC 降 低 ${ }^{[121-124]}$ 。此外，生物炭中的 $\mathrm{K} 、 \mathrm{Ca} 、 \mathrm{Mg}$ 等碱金属 增加, 生物炭“激发”的土壤微生物活动增强, 也可 能使生物炭 $\mathrm{CEC}$ 提高 ${ }^{[78,125]}$ 。

\section{5 吸附性}

生物炭极其丰富的多微孔结构、大比表面积, 使生物炭具有强吸附力。生物炭的吸附性能与其 多微孔结构、比表面积、表面官能团等有关, 亦受 到生产工艺、热解炭化温度、酸碱环境条件等诸

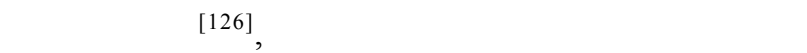
因子多、吸附过程较为复杂 ${ }^{[127]}$ 。生物炭所具有的 吸附性，使其可广泛用于重金属、有机污染物、有 害气体等不同介质中的污染物防控, 并可作为吸 附剂、载体或基质等功能材料广泛应用于农业、 环境、化工等领域，应用潜力、空间巨大 ${ }^{[97,40]}$ 。目 前, 关于生物炭吸附性的研究较多, 涉及领域多、 范围广，有关介质、吸附条件、作用因子、吸附过 程等较为繁杂, 在此不再赘述。以下仅就生物炭在 农业、环境等领域应用的基本吸附原理、过程等 作以简述。

环境领域。作为吸附功能材料, 利用生物炭进 行重金属污染修复的研究较为常见，其基本吸附过 程如图 2-a 所示, 主要包括静电吸引、离子交换、物 理吸附、表面络合和/或沉淀等过程 ${ }^{[87]}$ 。生物炭对重 金属的吸附作用, 主要源于生物炭表面所含有的丰 富含氧基团对重金属的强吸附作用 ${ }^{[87]}$, 而生物炭的 矿物成分在吸附过程中也具有至关重要的作用, 不 仅可为重金属吸附提供结合位点 ${ }^{[114]}$, 而且可通过提 高生物炭 $\mathrm{pH}$ ，降低有效态重金属离子活性、促进重 


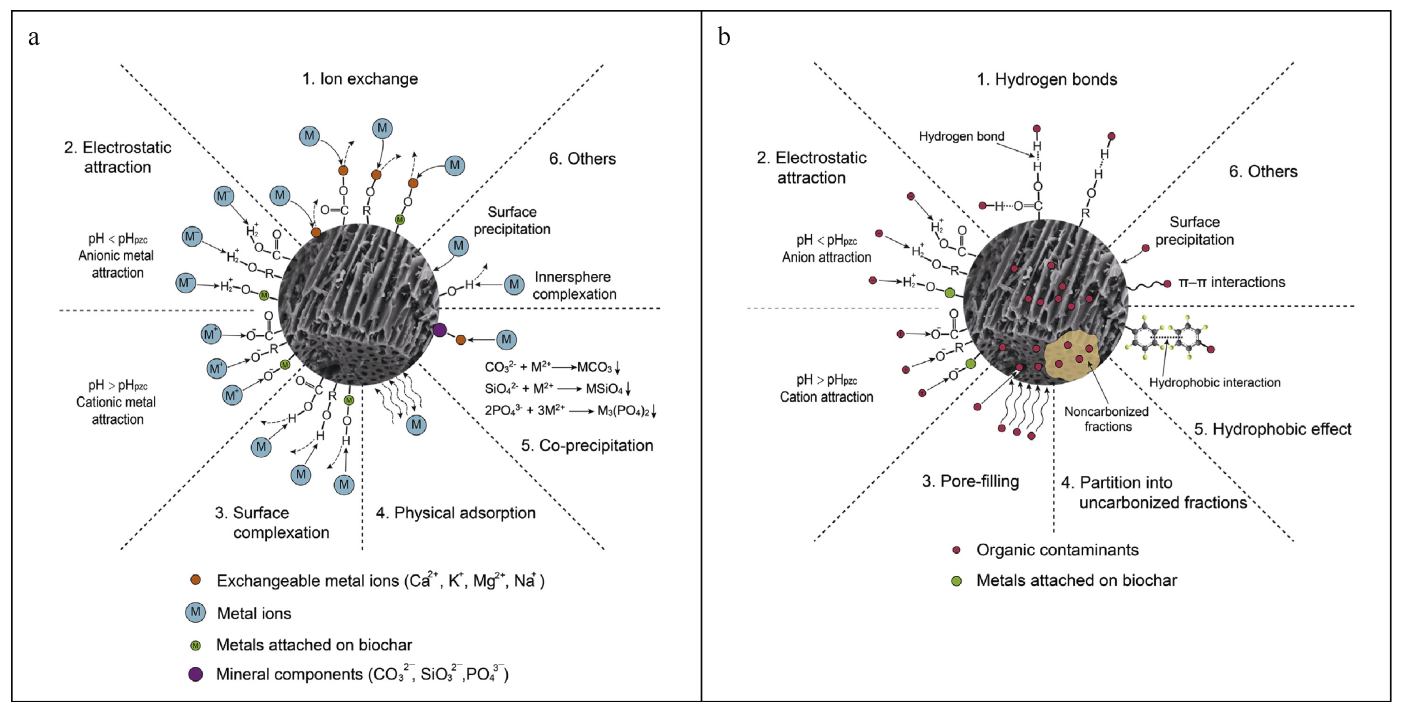

图 2 生物炭对重金属(a)和有机污染物(b)吸附模型 ${ }^{[87]}$

Fig. 2 Summary of mechanisms for heavy metals (a) and organic contaminants (b) adsorption on biochars ${ }^{[87]}$

金属沉淀，从而减少土壤中重金属浸出、降低重金 属生物有效性 ${ }^{[128]}$ 。与此同时, 生物炭极其丰富的多 微孔结构也会增强其对重金属离子的吸附、拦截, 从而降低重金属离子活性及其移动性, 并可能使重 金属形态发生改变 ${ }^{[129]}$ 。

农业领域。现实农业生产中，过量化肥、农药 施用等造成的面源污染日益突出, 已成为制约农业 “低碳、绿色、可持续”发展的“瓶颈”。研究表明, 生 物炭对降低农药等有机污染物残留, 减少土壤中过 量化肥养分流失等农业面源污染问题具有重要作 用 ${ }^{[130]}$ 。生物炭对有机污染物的吸附作用如图 2-b 所 示, 主要包括静电作用、疏水作用、氢键、 $\pi-\pi$ 作用、 孔隙填充等作用过程。生物炭对有机污染物的吸附, 主要源于生物炭中未炭化部分生物质的分配作用和 炭化部分的吸附作用 ${ }^{[87]}$, 其中非炭化部分的分配作 用是一个线性、非竞争性的吸附过程 ${ }^{[127]}$ ，而炭化部 分的吸附作用具有非线性等温线、共存吸附质间竞 争的特性。生物炭对有机污染物的吸附作用过程不
同, 主要与在相对较低炭化温度条件下 $\left(<700^{\circ} \mathrm{C}\right)$, 部分生物质未完全碳化 ${ }^{[100,131]}$, 使生物炭含有炭化/ 未炭化部分有关。在实际应用过程中, 生物炭对有 机污染物的吸附一般为多种吸附作用过程的塊联、 结合, 较为复杂 ${ }^{[87]}$ 。在农业生产中, 生物炭可作为基 质、载体或吸附剂材料, 用于吸附或固持 $\mathrm{N} 、 \mathrm{P} 、 \mathrm{~K}$ 等养分离子，减少土壤养分流失，水体“富营养化” 污染净化, 以及提高作物养分利用效率等 ${ }^{[97]}$, 其吸 附作用与生物炭多微孔构造、表面官能团、比表面 积及离子交换性能等有关 ${ }^{[97,132]}$ 。

\section{6 疏水/持水性}

生物炭特殊的结构和理化特性使其具有一定 疏水/持水性(图3) ${ }^{[133]}$ 。生物炭具有疏水性，与其在 炭化过程中的表面含氧官能团减少有关 ${ }^{[8]}$ 。而生物 炭具有持水性, 主要源于其丰富的多微孔结构增 加了对水的吸附力 ${ }^{[134]}$ 。生物炭持水性能主要取决 于其疏水/持水性在水分吸附上的抵消、平衡或叠 加作用 ${ }^{[119]}$ 。
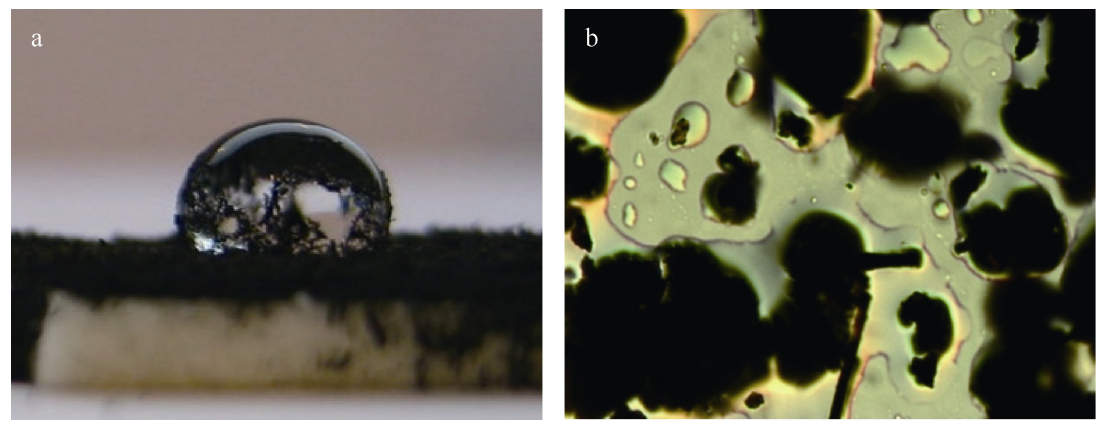

图 3 生物炭的疏水性(a)和持水性(b) ${ }^{[133]}$

Fig. 3 Hydrophobicity (a) and water holding capacity (b) of biochar ${ }^{[133]}$ 
生物炭的疏水性是其作为非溶性吸附质的重要 基础, 其性能大小可通过测定其含 $\mathrm{O} 、 \mathrm{~N}$ 官能团数量 来表征, 含 $\mathrm{O} 、 \mathrm{~N}$ 官能团数量越低、疏水性越强 ${ }^{[8]}$ 。 生物炭的疏水性与炭化温度密切相关, 炭化温度越 高、疏水性越强, 与生物炭表面极性官能团减少、 芳香性增加有关 ${ }^{[45,50,135]}$ 。生物炭的持水性则与其多 孔构造及不同炭化温度下的孔隙连通性有关 ${ }^{[119]}$, 在 低温炭化条件下, 生物炭的孔径较小、互连性较低, 产生的焦油成分易堵塞孔隙, 导致其持水性能下 降 ${ }^{[136]}$, 而在高温炭化条件下, 生物炭的多微孔结构 及数量增加、孔隙连通性增强, 对水分的物理容纳 和吸附力增强, 使其持水性能提高 ${ }^{[119,137]}$ 。

\section{7 稳定性}

生物炭含有较高的 C, 具有稳定的芳香族碳结 构, 使其具有较高稳定性 ${ }^{[138]}$ 。生物炭结构中的芳环 凝聚程度, 影响生物炭功能的稳定性和持久性, 而 非芳香族结构/官能团则有利于形成簇状结构, 也对 生物炭稳定性产生一定影响 ${ }^{[37]}$ 。生物炭的稳定性, 通常采用 $\mathrm{H} / \mathrm{C} 、 \mathrm{O} / \mathrm{C}$ 表征 ${ }^{[139]}$ 。低H/C和O/C比, 表明 生物炭具有较高熔融芳香环结构、稳定性较强, 反 之亦然 ${ }^{[140]}$ 。一般认为, 生物炭的 $\mathrm{O} / \mathrm{C}$ 摩尔比小于 0.2 被认为是最稳定的, 具有超千年的半衰期, $\mathrm{O} / \mathrm{C}$ 比在 $0.2 \sim 0.6$ 之间的生物炭半衰期在 100 1000年之间, 而 $\mathrm{O} / \mathrm{C}$ 比大于 0.6 的生物炭半衰期小于 100 年 ${ }^{[141]}$ 。

原料类型、炭化温度是影响生物炭稳定性的主

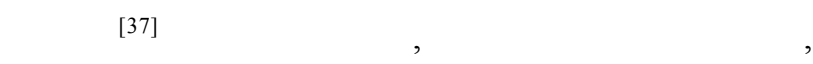
其次是纤维素和半纤维素 ${ }^{[142]}$, 原料中的木质素含量 越高, 炭化形成的芳香族 $\mathrm{C}$ 含量越高, 生物炭的稳 定性越强 ${ }^{[90]}$ 。因此, 木质纤维素类生物炭比非木质 纤维素类生物炭更稳定, 抗生物降解的能力更强 ${ }^{[40]}$ 。

原料中的金属、金属化合物或矿物等组分会在一定 程度上降低生物炭的稳定性 ${ }^{[143]}$, 但一些碱金属、碱 土金属及硅等元素会增强生物炭的稳定性, 如生物 炭中的非晶硅与 $\mathrm{C}$ 相互作用形成稳定的 Si-C, 从而 防止 $\mathrm{C}$ 被氧化, 使生物炭稳定性增强 ${ }^{[144]}$ 。此外, 原 料尺寸也会在一定程度上影响生物炭的稳定性, 一 般大颗粒/尺寸原料制备的生物炭稳定性高于小颗 粒/尺寸原料制备的生物炭 ${ }^{[145-146]}$, 其主要原因在于 大颗粒/尺寸原料在热解炭化时, 气相与固相物质之 间的接触时间更长, 聚合形成更多的碳, 从而使生 物炭固定碳含量提高、稳定性增强 ${ }^{[139]}$ 。热解炭化温 度, 则决定生物炭的芳香性及芳香凝聚度, 生物炭 中的大部分不稳定组分会随着炭化温度升高而逐渐
消失, 熔融芳香环结构增加, 不稳定的非芳香环结 构、大小及数量呈下降趋势 ${ }^{[37]}$ 。此外, 加热速率也 会对生物炭稳定性产生一定影响, 加热速率慢利于 增强生物炭稳定性 ${ }^{[147]}$ 。

综上, 生物炭的原料来源丰富, 不以消耗不可 再生资源和损害生态环境为代价，具有“低成本、可 再生、可持续”的突出优势，且制备过程“低碳、环保”, 利于促进资源与环境的“循环、永续”发展 ; 生物炭 的“富碳”多微孔结构使其可作为载体、基质等“构架 性”功能材料，从而发挥稳定的“结构重建”或“构相 改良”作用，而生物炭呈碱性、含碳量高、比表面积 大、吸附力强, 富含多种养分、表面官能团等多种 特性, 则使其可在固碳减排、改土培肥、防控污染、 节肥增效、促长增产等方面发挥综合性“叠加”效应, 其“多向、多效”增益及“累积、持续”性功效作用突 出; 生物炭独特的来源、结构、特性及功效优势, 使 其有别于其他同类材料, 具有显著经济、生态和社 会效益, 发展潜力和应用空间巨大。

\section{5 生物炭理化特性调控技术进展}

生物炭的理化特性, 是其功效发挥的“根源”, 重要性不言而喻。国内外研究者从不同学科、领域 和研究背景出发, 采用不同技术与方法, 针对生物 炭理化特性调控技术开展了积极、有益的科学探索, 取得了一定研究进展 ${ }^{[148]}$ 。目前, 主要的调控技术途 径: 1)在“前、中、后”炭化过程中添加外源物质, 使 其与原料或炭化产物发生理化反应, 进而改变生物 炭表面或内部结构及特性, 从而获得某些具有特定 性质或功能的生物炭材料; 2)通过调控、优化炭化 工艺及制备方法, 对生物炭结构及理化性质进行调 控, 使生物炭构相、特性发生某些定向或优化改变, 从而达到预期应用目的和目标。在实际运用过程中, 一般多采用上述两种或二者相结合的技术途径。

\section{1 外源介质添加改性技术}

生物炭具有良好的多微孔、吸附性及稳定性, 但在某些特定用途、目标条件下还不能满足现实 需求。因此, 一些研究者尝试在炭化过程中, 通过 添加氧化剂、磁化物等外源介质, 使其与生物炭发 生一些物理-化学反应, 重塑炭表面、内部某些结 构及特性, 使之达到目标特性最优化 ${ }^{[149-150]}$ 。目前, 主要有磁化改性、氧化剂改性、涂层/浸渍改性等 调控技术。

\section{1 .1 磁化改性在污水净化、重金属污染修复}


等环保领域，生物炭的使用需求和应用空间巨大。 但是, 由于生物炭质轻、密度低, 不易与水等介质分 离 ${ }^{[154]}$, 使生物炭的应用空间和量级受限。因此, 一 些研究者通过将生物炭与磁性介质结合, 在外磁场 控制下实现污水固、液分离 ${ }^{[151-153]}$, 取得了一定效果 和进展。一般情况下, 磁性介质多采用铁或铁氧化 物, 如铁 $(0) 、 \gamma-\mathrm{Fe}_{2} \mathrm{O}_{3} 、 \mathrm{Fe}_{3} \mathrm{O}_{4} 、 \mathrm{CoFe}_{2} \mathrm{O}_{4}$ 等 ${ }^{[148]}$ 。改性 后的磁化生物炭, 阳离子交换量、孔隙数量等大幅 提高, 对污染物的吸附、去除能力明显增强 ${ }^{[155-156]}$ 。 研究发现, 磁化改性生物炭对水体中的重金属离 子、有机污染物等具有较强吸附作用, 其整体吸附 性能高于非磁化生物炭 ${ }^{[148,155]}$ 。磁化改性技术的应用, 进一步扩展了生物炭的应用空间, 实用性、易用性 提高。

\section{1 .2 氧化剂改性氧化剂改性, 一般采用化学} 氧化剂为介质, 使其与生物炭发生氧化反应, 改变 生物炭的结构、酸碱性、官能团、比表面积等特性, 增强其吸附等性能, 从而提高生物炭对土壤、水体 中污染物的吸附、去除效率 ${ }^{[157]}$ 。一般情况下，氧化 剂改性多采用过氧化氢、强酸或强碱类化学剂。

过氧化氢改性, 其优点是成本低, 可避免与其 他元素产生干扰，适于土壤改良、水体净化等应用 场景 ${ }^{[148]}$ 。研究发现, 采用过氧化氢处理的改性生物 炭, 其表面含氧官能团数量大幅增加、吸附能力明 显提高 ${ }^{[158-159]}$ 。强酸类氧化剂改性，一般采用硫酸、 硝酸等化学试剂为介质。研究表明, 当炭化温度达 到 $700^{\circ} \mathrm{C}$ 时, 以硫酸为介质氧化处理后的改性生物 炭, 其比表面积显著提高, 是未活化改性前的 250 倍以上 ${ }^{[160]}$ 。采用硝酸改性, 会造成生物炭的结构性 坍塌和 $\mathrm{pH}$ 降低、比表面积减小, 但会增加生物炭的 表面酸性基团数量, 提高其表面亲水性 ${ }^{[148,161]}$ 。强碱 类氧化剂改性, 一般采用氢氧化钠、氢氧化钾等化 学剂。研究发现, 采用氢氧化钠改性, 会显著提高生 物炭的表面积和微孔体积, 提高其对重金属离子的 吸附能力 ${ }^{[162-164]}$ 。而采用氢氧化钾改性, 生物炭的微 孔数量、吸附性能也有一定程度提高 ${ }^{[165-166]}$ 。

\section{1 .3 涂层/浸渍改性一般指在炭化前或炭化}

后，采用涂层或浸渍方法，将外源金属氧化物、有机 试剂、纳米材料等介质与生物炭进行物理或化学反 应耦合, 从而使生物炭比表面积、多孔性、表面官 能团、阳离子交换量等特性改变的方法 ${ }^{[148,167]}$ 。

采用金属氧化物涂层改性，可在不同程度上提
高改性生物炭的吸附能力 ${ }^{[168-169]}$ ，当浸有金属离子 的生物质在炭化后，其所含有的金属离子形成金属 氧化物或氢氧化物, 成为负载金属的炭基复合材 料 ${ }^{[148]}$ 。研究发现, 与未改性生物炭相比, 钴涂层改 性生物炭具有更高的表面积、孔体积，对铬离子的 吸附量显著增加 ${ }^{[169]}$, 而采用 $\mathrm{MgCl}_{2}-6 \mathrm{H}_{2} \mathrm{O}$ 和 $\mathrm{AlCl}_{3}$ $6 \mathrm{H}_{2} \mathrm{O}$ 与生物炭复合制备的炭基复合材料, 对磷的最 大吸附量提高了 5 50倍 ${ }^{[170]}$ 。此外, 有研究采用聚乙 烯亚胺和戊二醛与生物炭复合, 发现改性后的生物 炭含氧官能团数量明显增加, 对 $\mathrm{Cd}^{6+}$ 的吸附性能明 显提高, 最大吸附量是未改性生物炭的 18.87 倍 ${ }^{[171]}$ 。 而采用乙二胺、三甲胺等有机试剂作为介质, 改性 后的生物炭孔隙结构更为发达、表面基团数量明显 增加，对硝酸盐的吸附、去除效能提高 ${ }^{[172]}$ 。

近年来, 纳米材料的技术开发与应用得到了专 家、学者的广泛关注。但由于纳米材料颗粒小，易 团聚、氧化，在一定程度上限制了其应用 ${ }^{[173-174]}$ 。但 如果将纳米材料通过预涂(炭化前)和浸渍(炭化后) 等过程负载于生物炭表面，制备成功能性炭基纳米 复合材料, 可提供更多高亲和力吸附位点, 从而有 效发挥纳米材料的优势, 改善生物炭的多孔结构及 比表面积、官能团、热稳定性等特性(图4) ${ }^{[175]}$ 。研 究认为, 采用预涂、浸渍等处理方法, 将纳米材料 与生物炭结合, 可弥补高温热解后生物炭表面官能 团减少等“缺陷”，实现“纳米-生物炭”功能复合，充 分发挥纳米材料与生物炭材料的双重功效优势, 提 高其对污染物的吸附、去除效能 ${ }^{[176]}$ 。目前，采用涂 层/浸渍改性的纳米/高分子复合材料主要为石墨 烯、碳纳米管、壳聚糖等, 具有良好的应用潜力和 发展空间。

\section{2 炭化工艺调控技术}

炭化工艺及制备方法，决定了生物炭的结构 及主要理化特性。通过改进、优化和创新生物质 炭化工艺及其制备方法, 实现对生物炭结构及特 性的总体性、批量化调控，是工程化、规模化开发 生物炭功能材料及产品的必由之路。目前, 炭化工 艺调控技术主要有气体活化、微波炭化、球磨、 紫外辐照等。

5.2 .1 气体活化 气体活化, 一般指在一定炭化 温度条件下，采用水蒸气、二氧化碳等进行活化改 性的方法 ${ }^{[148]}$ 。通过气体活化技术, 可将常规生物炭 改性为结构更为丰富、比表面积更大的活性生物炭 


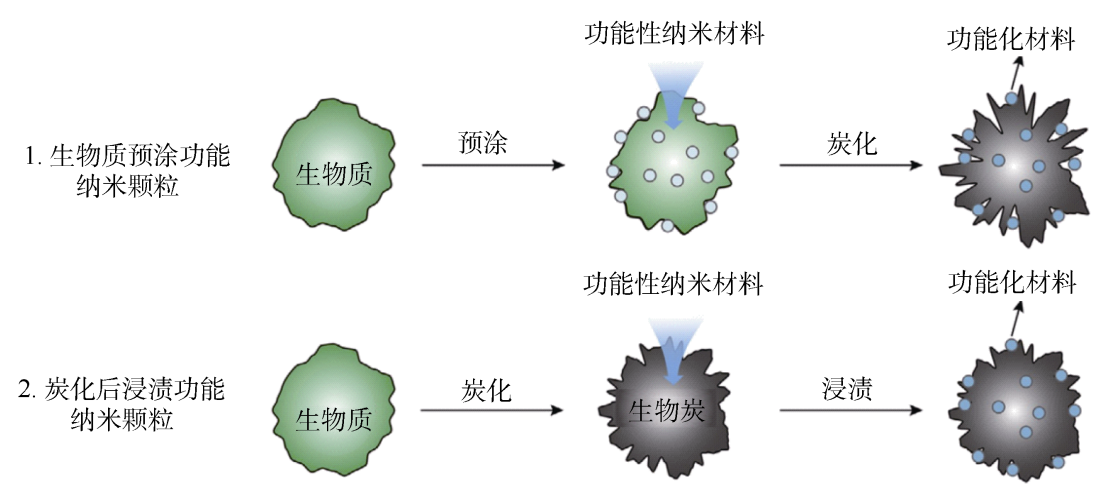

图 4 生物炭基纳米复合材料的合成制备过程 ${ }^{[175]}$

Fig. 4 Schematic diagram of synthesizing biochar-based nano-composites ${ }^{[175]}$

材料 ${ }^{[177]}$ 。研究发现, 在 $700^{\circ} \mathrm{C}$ 条件下采用蒸汽活化 的生物炭比未活化生物炭的表面积提高了一倍 ${ }^{[178]}$, 吸附性能显著提升。

气体活化改性生物炭, 多用于水体净化、污染 物处理等领域 ${ }^{[179-181]}$ 。研究发现, 经特定蒸汽流量活

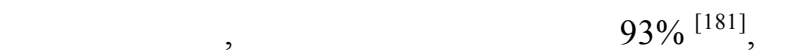
泉离子也表现良好吸附性 ${ }^{[182]}$ 。另有研究表明, 与未 活化生物炭相比, 蒸汽活化生物炭可提高其对土壤 氮、磷等养分离子的固持能力, 减少养分流失 ${ }^{[183]}$ 。 气体活化改性是一种简单、有效的改性技术方法, 但由于生物炭非均质性强, 反应温度、活化程度等 条件难以精确控制, 因而可能造成对生物炭活化不 均、局部过热等问题, 影响生物炭的活化质量及其 功能作用, 在工业化生产中尚需进行深入的工艺与 技术创新 ${ }^{[184]}$ 。

\subsection{2 微波炭化微波, 是一种频率在 300 兆赫}

至 300 千兆赫之间的高频电磁波, 可迅速穿透生物 质并将能量传递给反应物官能团 ${ }^{[185]}$ 。采用微波改性 方法制备的生物炭, 在官能团数量、比表面积及稳 定性等方面优于传统热解方法制备的生物炭 ${ }^{[186-187]}$ 。 研究表明, 微波改性生物炭可提高其对重金属污染 物的吸附、去除效率 ${ }^{[188-190]}$ 。另有研究通过在微波制 炭过程中添加特定化学物质, 使生物炭与特定反应 物在微波条件下发生物理-化学反应, 也取得了良好 试验效果 ${ }^{[191]}$ 。未来, 在精细化、多元化应用目标趋 动下, 在微波裂解过程中添加化学改性剂或其他特 性材料, 已成为一种发展趋势 ${ }^{[148]}$ 。

\subsection{3 球磨制炭球磨制炭, 一般指采用球磨仪} 对生物炭材料进行机械球磨, 降低其固体颗粒粒度, 从而改变生物炭结构, 提高生物炭整体性能的方 法 ${ }^{[192]}$ 。球磨后的生物炭颗粒大小可达纳米级, 比表 面积、吸附性能显著提高, 去除有机、无机污染物
性能表现与碳纳米管相当 ${ }^{[193]}$ 。由于球磨生物炭的颗 粒更小, 使其比表面积更大, 利于增加对有机、无机 离子的潜在吸附位, 使球磨生物炭具有优异的整体 吸附性能 ${ }^{[194-195]}$ 。经球磨优化条件下制备的生物炭, 其表面积增加 60 194 $\mathrm{m}^{2} \mathrm{~g}^{-1}$, 吸附性能显著提高 ${ }^{[196]}$ 。

但是, 由于球磨生物炭在水中的分散性强、不 易控制，在一定程度上限制了其在环保等领域的应 用。此外, 由于球磨生物炭的颗粒较轻, 易发生地表 径流侵蚀和场外输送, 因而可能造成潜在的生态安 全风险。研究表明, 随着生物炭粒径减小, 生物炭迁 移量明显增加 ${ }^{[197]}$ 。生物炭胶体粒子的移动, 可能导 致农药及其他污染物沿土壤剖面发生非现场迁移, 从而增加地下水的潜在环境安全风险 ${ }^{[198]}$ 。目前, 在 球磨生物炭的结构及特性研究方面, 已取得一定研 究进展, 但受机械水平、产能及应用条件、范围、 生态安全性等因素影响, 距“工程化、规模化”开发与 应用还有一定距离。

5.2 .4 紫外辐照紫外辐照, 一般指采用一定波 段的紫外光进行辐照改性的方法。紫外光辐照改性 生物炭, 其比表面积、含氧官能团数量明显提高, 对 $\mathrm{Pb}^{2+} 、 \mathrm{Cd}^{2+}$ 金属离子的吸附能力明显增强, 最大吸附 量显著提升 ${ }^{[199-200]}$ 。紫外辐照改性方法, 操作简单、 环保、安全, 为生物炭改性技术发展提供了新途径。 但是, 由于实施紫外辐照的条件、容量和规模等因 素制约，使其在“规模化、工程化”及“精准性、稳定 性”等方面，还存在一定局限性。

生物炭结构及理化特性, 决定了其作为载体、 基质或吸附剂等功能材料的“适用、实用和经济性”, 采用新材料、新工艺、新方法对生物炭构相及其理 化特性进行“定性、定量化”调控, 是一种必然趋势。 目前, 生物炭改性技术已成为生物炭领域的研究热 点之一，正由单一技术转向 “ $1+\mathrm{N}$ ”复合技术，由原 
始、传统工艺转向“精准化、智能化、自动化”现代 新工艺, 是目前改性技术的发展趋势, 生物炭改性 技术的创新发展与应用有望为生物炭基础研究与应 用提供新方向、新途径、新突破。

\section{6 研究展望}

时至今日, 生物炭在农业、环境、能源等领域 所展现的重要功能、潜在价值与贡献, 已得到全球 专家、学者的广泛关注和研究认可。我国在陈温福 院士等一批科学家的倡导和努力下, 在生物炭制备 工艺、基础研究、应用技术、产品开发及产业化等 方面所取得的研究进展与成果, 已处于国际前列。 生物炭研究“方兴末艾”, 已步入“快车道”, 多领域、 多学科先进技术的创新与发展, 必将推动生物炭研 究与应用向“大范围、宽领域”、“综合性、交叉性” 和“定制化、多元化”方向快速发展。开展生物炭特 性及其应用研究，在以下几个方面值得关注:

\section{1 研究方向}

（1）面向我国农业发展国情，结合区域农业发 展特征, 突破“低成本、高效、环保”炭化工艺瓶颈, 发展“小、中、大”兼具、“炭、油、气”联产, 适合不 同农林废弃物来源的炭化生产系统，建立“分散-集 中”式、多向覆盖的炭化生产网络, 依靠科技创新扩 大产能、降低生产与流通成本，使炭化工艺技术、 装备与产品真正“落地、触底”，真正“可用、能用、 适用”。

（2）基于农、林废弃物“循环、高效”利用，聚焦 农业、环境等领域“老、大、难”生产现实问题，采用 原创或交叉、集成创新技术，深入挖掘生物炭“构效”与“质-效”作用潜力及优势，重点开发利于自然 生态系统“平衡、稳定、永续”发展，真正“低碳、生 态、高效”的普适、多元、高值化系列炭基功能材料 及产品。

(3) 建立“科学、系统、规范”的生物炭生产、测 定与应用指标评价体系, 使生物炭研究与应用结果 科学、准确, 有依据、可比较; 创建基于不同来源、 材质、工艺等条件下的生物炭理化特性与应用数据 库, 使生物炭研究与应用“可查、可溯、可依”, 为相 关研究、使用者提供有效参考; 制定生物炭材料、 产品及其应用等相关技术标准，规范生物炭来源、 生产、制备及应用等各环节, 破解生物炭制备、应 用及市场乱象, 促进生物炭产业“健康、稳定、有序” 发展。
(4) 生物炭研究与应用的“多领域融合、多学科 交叉、多功能复合”, 生物炭材料及其产品的“特色 化、功能化、定制化”，是其未来发展的趋势和方向； 未来, 生物炭基材料及产品很可能以“低碳、绿色、 环保”等综合功效优势，替代部分传统农业与环保投 入品，以“零资源损耗、低成本投入”，获得“多效、 稳定、可持续”收益, 实现经济、生态和社会效益最 大化，为农业、资源与环境的“循环、可持续”发展提 供“趋动力”。

\section{2 展望}

目前, 生物炭理化特性及应用研究已取得一定 进展, 但目前全球大多数研究仍处于实验室或模 拟、小规模试验条件下，距“批量化、规模化、工程 化”应用和实施还存在较大差距。在基础研究方面, 不同来源、材质、炭化工艺条件下生物炭的“构-效” 与“质-效”的定性、定量化关系，炭基复合、载体功 能材料制备及其应用的物理-化学反应过程、调控途 径及作用原理、机制，炭基功能材料的“可定制化” 载体构建，生物炭基材料及其产品应用的“长期性、 安全性、可持续性”评估与预测等等, 还有诸多科学、 技术问题亟待研究探索 ; 在炭化工艺、装备及产品 研制方面，不同原料来源的“低成本、广适、环保” 型炭化新工艺，生物质炭化及其产品制备、生物炭 改性条件的“精准、定量”化控制，炭化副产物的“低 成本、零污染、高效”回收与再利用，改性生物炭的 “稳定性、可控性、安全性”等方面, 尚需开展系统、 深入的研究探索与实践; 纳米等创新技术发展, 为 炭基多功能、复合材料开发提供了新方向，在炭基 复合、介质材料的篮选、开发与应用，复合技术的“精 准、定量化”控制, 以及炭基多功能、复合材料与产 品的“批量化、规模化”生产与应用等方面还有较大 提升空间;打破传统技术的“单一性”桎梏，融合多领 域、多学科交叉技术，探索“生物炭+”技术模式，是 未来突破末来生物炭特性与功能局限, 研发新一代 “复合性、功能化、高效型”炭基材料及产品的发展 重要方向; “多元化、功能化、定制化”是未来生物 炭改性技术及其产品开发的发展趋势, 有望在解决 农业、环境等领域突出问题中发挥重要支撑性作用。

科学需要理性, 更应该理性对待科学。作为新 生事物, 有关于生物炭的定义、结构、功效及其应 用等方面还存在不同观点。但不可否认的是, 生物 炭在农业、环境等领域应用确有着良好的综合效益 及重要作用潜力、价值与贡献, 其独特的来源、结 
构、特性及功效是其他材料所不具备的, 也得到了 全世界科学家的普遍关注和一致研究认可。当前, 我国正处于农业与经济、社会发展的战略转型期, 生物炭无疑为解决农林废弃物综合利用难题, 促进 资源与环境“循环、永续”发展, 提供了重要、可行性 新途径, 为保障国家粮食安全、提升耕地质量, 促进 “三农”发展提供有效技术支撑。相信, 未来在国内外 专家、学者的共同努力下, 生物炭的研究与应用发 展及其产业化实施, 必将为人类面对气候变化, 破 解“沃土、碧水、蓝天”可持续发展问题与矛盾, 打造 “绿水、青山”, 作出应有的重要贡献。

\section{References}

[1] Bapat H, Manahan S E, Larsen D W. An activated carbon product prepared from milo (Sorghum vulgare) grain for use in hazardous waste gasification by ChemChar concurrent flow gasification. Chemosphere, 1999, 39: 23-32.

[2] Lehmann J, Gaunt J, Rondon M. Bio-char sequestration in terrestrial ecosystems: a review. Mitig Adapt Strat Gl, 2006, 11: 403-427.

[3] Glaser B, Lehmann J, Zech W. Ameliorating physical and chemical properties of highly weathered soils in the tropics with charcoal: a review. Biol Fert Soils, 2002, 35: 219-230.

[4] Lehmann J, Joseph S. Biochar for Environmental Management: Science, Technology and Implementation, 2nd edn. London: Earthscan from Routledge, 2015. pp 1-1214.

[5] International Biochar Initiative (IBI). International Biochar Initiative-Standardized Product Definition and Product Testing Guidelines for Biochar That Is Used in Soil (aka IBI Biochar Standards) Version 2.0. International Biochar Initiative: Westerville, OH, USA, 2014.

[6] Zhang Z K, Zhu Z Y, Shen B X, Liu L N. Insights into biochar and hydrochar production and applications: a review. Energy, 2019, 171: 581-598.

[7] Li S, Harris S, Anandhi A, Chen G. Predicting biochar properties and functions based on feedstock and pyrolysis temperature: a review and data syntheses. J Clean Prod, 2019, 215: 890-902.

[8] Masebinu S O, Akinlabi E T, Muzenda E, Aboyade A O. A review of biochar properties and their roles in mitigating challenges with anaerobic digestion. Renew Sust Energ Rev, 2019, 103: 291-307.

[9] 陈温福, 张伟明, 孟军, 徐正进. 生物炭应用技术研究. 中国 工程科学, 2011, 13(2): 83-89.

Chen W F, Zhang W M, Meng J, Xu Z J. Researches on biochar application technology. Eng Sci, 2011, 13(2): 83-89 (in Chinese with English abstract).

[10] Azargohar R, Jacobson K L, Powell E E, Dalai A K. Evaluation of properties of fast pyrolysis products obtained, from Canadian waste biomass. J Anal Appl Pyrol, 2013, 104: 330-340.

[11] Maschio G, Koufopanos C, Lucchesi A. Pyrolysis, a promising route for biomass utilization. Bioresour Technol, 1992, 42: 219-231.

[12] Zhang L H, Xu C, Champagne P. Overview of recent advances in thermo-chemical conversion of biomass. Energ Convers Manage, 2010, 51: 969-982.

[13] McGrath T E, Chan W G, Hajaligol M R. Low temperature mechanism for the formation of polycyclic aromatic hydrocarbons from the pyrolys is of cellulose. J Anal Appl Pyrol, 2003, 66: 51-70.

[14] Roy P, Dias G. Prospects for pyrolysis technologies in the bioenergy sector: a review. Renew Sust Energ Rev, 2017, 77: 59-69.

[15] Rangabhashiyam S, Balasubramanian P. The potential of lignocellulosic biomass precursors for biochar production: performance, mechanism and wastewater application: a review. Ind Crop Prod, 2019, 128: 405-423.

[16] Li D C, Jiang H. The thermochemical conversion of non-lignocellulosic biomass to form biochar: a review on characterizations and mechanism elucidation. Biores Technol, 2017, 246: $57-68$.

[17] Vaibhav D, Thallada B. A comprehensive review on the pyrolysis of lignocellulosic biomass. Renew Energy, 2017, 129: 695-716.

[18] Huber G W, Iborra S, Corma A. Synthesis of transportation fuels from biomass: chemistry, catalysts, and engineering. Chem Rev, 2006, 106: 4044-4098.

[19] Yu J, Paterson N, Blamey J, Millan M. Cellulose, xylan and lignin interactions during pyrolysis of lignocellulosic biomass. Fuel, 2017, 191: 140-149.

[20] Sonil N, Javeed M, Sivamohan N R, Janusz A K, Ajay K D. Pathways of lignocellulosic biomass conversion to renewable fuels. Biomass Convers Bior, 2014, 4: 157-191.

[21] Gallezot P. Conversion of biomass to selected chemical products. Chem Soc Rev, 2012, 41: 1538-1558.

[22] Toor S S, Rosendahl L, Rudolf A. Hydrothermal liquefaction of biomass: a review of subcritical water technologies. Energy, 2011, 36: 2328-2342.

[23] Shen D K, Gu S, Bridgwater A V. Study on the pyrolytic behaviour of xylan-based hemicellulose using TG-FTIR and Py-GC-FTIR. J Anal Appl Pyrol, 2010, 87: 199-206.

[24] Liu W J, Jiang H, Yu H Q. Development of biochar-based functional materials: toward a sustainable platform carbon material. Chem Rev, 2015, 115: 12251-12285.

[25] Li S, Lyons-Hart J, Banyasz J, Shafer K. Real-time evolved gas analysis by FTIR method: an experimental study of cellulose pyrolysis. Fuel, 2001, 80: 1809-1817.

[26] Cao X F, Sun S N, Sun R C. Application of biochar-based catalysts in biomass upgrading: a review. Rsc $A d v, 2017,7$ : 48793-48805.

[27] Kosa M, Ben H, Theliander H, Ragauskas A J. Pyrolysis oils from $\mathrm{CO}_{2}$ precipitated Kraft lignin. Green Chem, 2011, 13: 3196.

[28] Zhang J, Nolte M W, Shanks B H. Investigation of primary reactions and secondary effects from the pyrolysis of different celluloses. ACS sustain. Chem Eng, 2014, 2: 2820-2830.

[29] Morf P, Hasler P, Nussbaumer T. Mechanisms and kinetics of homogeneous secondary reactions of tar from continuous pyrolysis of wood chips. Fuel, 2002, 81: 843-853.

[30] Wei L, Xu S, Zhang L, Zhang H, Liu C, Zhu H, Liu S. Characteristics of fast pyrolysis of biomass in a free fall reactor. Fuel Process Technol, 2006, 87: 863-871.

[31] Tsai W T, Lee M K, Chang J H, Su T Y, Chang Y M. Characteri- 
zation of bio-oil from induction-heating pyrolysis of food-processing sewage sludges using chromatographic analysis. Bioresour Technol, 2009, 100: 2650-2654.

[32] Pokorna E, Postelmans N, Jenicek P, Schreurs S, Carleer R, Yperman J. Study of bio-oils and solids from flash pyrolysis of sewage sludges. Fuel, 2009, 88: 1344-1350.

[33] Shaheen S M, Niazi N K N, Hassan N E E, Bibi I, Wang H L, Tsang D C W, Ok Y S, Bolan N, Rinklebe J. Wood-based biochar for the removal of potentially toxic elements in water and wastewater: a critical review. Int Mater Rev, 2019, 64: 216-247.

[34] Chen W F, Meng J, Han X R, Lan Y, Zhang W M. Past, present, and future of biochar. Biochar, 2019, 1: 75-87.

[35] Liu Y X, Lonappan L, Brar S K, Yang S M. Impact of biochar amendment in agricultural soils on the sorption, desorption, and degradation of pesticides: a review. Sci Total Environ, 2018, 645: $60-70$.

[36] Warnock D D, Lehmann J, Kuyper T W, Rillig M C. Mycorrhizal responses to biochar in soil-concepts and mechanisms. Plant Soil, 2007, 300: 9-20.

[37] Leng L J, Huang H J. An overview of the effect of pyrolysis process parameters on biochar stability. Bioresour Technol, 2018, 270: 627-642.

[38] Qambrani N A, Rahman M M, Wonc S, Shima S, Ra C. Biochar properties and eco-friendly applications for climate change mitigation, waste management, and wastewater treatment: a review. Renew Sust Energ Rev, 2017, 79: 255-273.

[39] 袁金华, 徐仁扣. 生物质炭的性质及其对土壤环境功能影响 的研究进展. 生态环境学报, 2011, 20: 779-785.

Yuan J H, Xu R K. Progress of the research on the properties of biochars and their influence on soil environmental functions. Soil Environ Sci, 2011, 20: 779-785 (in Chinese with English abstract).

[40] Chen Y Q, Yang H P, Wang X H, Zhang S H, Chen H P. Biomass-based pyrolytic polygeneration system on cotton stalk pyrolysis: influence of temperature. Biores Technol, 2012, 107: 411-418.

[41] Shaaban M, Zwieten L V, Bashir S, Younas A, Núñez-Delgado A, Chhajro M A, Kubar K A, Ali U, Rana M S, Mehmood M A, Hu R G. A concise review of biochar application to agricultural soils to improve soil conditions and fight pollution. J Environ Manage, 228: 429-440.

[42] Yang H, Yan R, Chen H, Lee D H, Zheng C. Characteristics of hemicellulose, cellulose and lignin pyrolysis. Fuel, 2007, 86: 1781-1788.

[43] Keiluweit M, Nico P S, Johnson M G, Kleber M. Dynamic molecular structure of plant biomass-derived black carbon (biochar). Environ Sci Technol, 2010, 44: 1247-1253.

[44] Joseph S D, Downie A, Crosky A, Lehmann J, Munroe P. Biochar for carbon sequestration, reduction of greenhouse gas emissions and enhancement of soil fertility: a review of the materials science. Rend Circ Mat Palermo Suppl, 2007, 48: 101-106.

[45] Cantrell K B, Hunt P G, Uchimiya M, Novak J M, Ro K S. Impact of pyrolysis temperature and manure source on physicochemical characteristics of biochar. Bioresour. Technol, 2012, 107: 419-428.

[46] Li H, Dong X, da Silva E B, de Oliveira L M, Chen Y, Ma L Q.
Mechanisms of metal sorption by biochars: biochar characteristics and modifications. Chemosphere, 2017, 178: 466-478.

[47] Liu Y X, Yao S, Wang Y Y, Lu H H, Brar S K, Yang S M. Bioand hydrochars from rice straw and pig manure: inter-comparison. Bioresour Technol, 2017, 235: 332-337.

[48] Halim S A, Swithenbank J. Characterisation of Malaysian wood pellets and rubberwood using slow pyrolysis and microwave technology. J Anal Appl Pyrol, 2016, 122: 64-75.

[49] Uzunova S, Angelova D, Anchev B, Uzunov I, Gigova A. Changes in structure of solid pyrolysis residue during slow pyrolysis of rice husk. Bulg Chem Commun, 2014, 46: 184-191.

[50] Chun Y, Sheng G G, Chiou C T, Xing B S. Compositions and sorptive properties of crop residue-derived chars. Environ Sci Technol, 2004, 38: 4649-4655.

[51] 黄华, 王雅雄, 唐景春, 朱文英. 不同烧制温度下玉米秸秆生 物炭的性质及对萗的吸附性能. 环境科学, 2014, 35: 1884-1890.

Huang H, Wang Y X, Tang J C, Zhu W Y. Properties of maize stalk biochar produced under different pyrolysis temperatures and its sorption capability to naphthalene. Environ Sci, 2014, 35: 1884-1890 (in Chinese with English abstract).

[52] Zhang J, Liu J, Liu R. Effects of pyrolysis temperature and heating time on biochar obtained from the pyrolysis of straw and lignosulfonate. Bioresour Technol, 2015, 176: 288-291.

[53] Qian K, Kumar A, Zhang H, Bellmer D, Huhnke R. Recent advances in utilization of biochar. Renew Sust Energ Rev, 2015, 42: $1055-1064$.

[54] 张伟明, 孟军, 王嘉宇, 范淑秀, 陈温福. 生物炭对水稻根 系形态与生理特性及产量的影响. 作物学报, 2013, 39: 1445-1451.

Zhang W M, Meng J, Wang J Y, Fan S X, Chen W F. Effect of biochar on root morphological and physiological characteristics and yield in rice. Acta Agron Sin, 2013, 39: 1445-1451 (in Chinese with English abstract).

[55] UC Davis Biochar Database, 2015. http://biochar.ucdavis.edu/ download/ (accessed 2016-12-25).

[56] Lehmann J. Bio-energy in the black. Front Ecol Environ, 2007, 5: 381-387.

[57] Novak J M, Lima I, Xing B, Gaskin J W, Steiner C, Das K C, Ahmedna M, Rehrah D, Watts D W, Busscher W J, Schomberg H. Characterization of designer biochar produced at different temperatures and their effects on a loamy sand. Ann Environ Sci, 2009, 3: 195-206.

[58] Spokas K A, Novak J M, Stewart C E, Cantrell K B, Uchimiya M, DuSaire M G, Ro K S. Qualitative analysis of volatile organic compounds on biochar. Chemosphere, 2011, 85: 869-882,

[59] Yuan J H, Xu R K. The amelioration effects of low temperature biochar generated from nine crop residues on an acidic Ultisol. Soil Use Manage, 2011, 27: 110-115.

[60] Xu X Y, Zhao Y H, Sima J, Zhao L, Mašek O, Cao X D. Indispensable role of biochar-inherent mineral constituents in its environmental applications: a review. Bioresour Technol, 2017, 101: 887-899.

[61] Al-Wabel M, Al-Omran A, El-Naggar A H, Nadeem M, Usman A R A. Pyrolysis temperature induced changes in characteristics and 
chemical composition of biochar produced from conocarpus wastes. Bioresour Technol, 2013, 131: 374-379.

[62] Wang Y, Hu Y, Zhao X, Wang S, Xing G. Comparisons of biochar properties from wood material and crop residues at different temperatures and residence times. Energ Fuel, 2013, 27: 5890-5899.

[63] Conti R, Rombolà A G, Modelli A, Torri C, Fabbri D. Evaluation of the thermal and environmental stability of switchgrass biochars by Py-GC-MS. J Anal Appl Pyrol, 2014, 110: 239-247.

[64] Angin D. Effect of pyrolysis temperature and heating rate on biochar obtained from pyrolysis of safflower seed press cake. Bioresour Technol, 2013, 128: 593-597.

[65] Tan Z X, Lin C S K, Ji X Y, Raineyd T J. Returning biochar to fields: a review. Appl Soil Ecol, 2017, 116: 1-11.

[66] Lehmann J, Pereira D S, Steiner C, Nehls T, Zech W, Glaser B. Nutrient availability and leaching in an archaeological a throsol and a ferralsol of central amazonia: fertilizer, and charcoal amendments. Plant Soil, 2003, 249: 343-357.

[67] 周劲松, 问平, 张伟明, 郑福余, 程效义, 陈温福. 生物炭对 东北冷凉区水稻秧苗根系形态建成与解剖结构的影响. 作物 学报, 2017, 43: 72-81.

Zhou J S, Yan P, Zhang W M, Zheng F Y, Cheng X Y, Chen W F. Effect of biochar on root morphogenesis and anatomical structure of rice cultivated in cold region of northeast China. Acta Agron Sin, 2017, 43: 72-81 (in Chinese with English abstract).

[68] Yavari S, Malakahmad A, Sapari N B. Biochar efficiency in pesticides sorption as a function of production variables: a review. Environ Sci Pollut R, 2015, 22: 13824-13841.

[69] Oliveira F R, Patel A K, Jaisi D P, Adhikaric S, Lu H, Khanala S K. Environmental application of biochar: current status and perspectives. Bioresour Technol, 2017, 246: 110-122.

[70] Chen Y Q, Zhang X, Chen W, Yang H P, Chen H P. The structure evolution of biochar from biomass pyrolysis and its correlation with gas pollutant adsorption performance. Bioresour Technol, 2017, 246: 101-109.

[71] Zhao B, O`Connor D, Zhang J, Peng T, Shen Z, Tsang D C W, Hou D. Effect of pyrolysis temperature, heating rate, and residence time on rapeseed stem derived biochar. J Clean Prod, 2018, 174: 977-987.

[72] Kim K H, Kim J, Cho T, Choi J W. Influence of pyrolysis temperature on physicochemical properties of biochar obtained from the fast pyrolysis of pitch pine (Pinus rigida). Bioresour Technol, 2012, 118: 158-62.

[73] Yao Y, Gao B, Chen H, Jiang L J, Inyang M, Zimmerman A R, Cao X D, Yang L Y, Xue Y W, Li H. Adsorption of sulfamethoxazole on biochar and its impact on reclaimed water irrigation. $J$ Hazard Mater, 2012, 209: 408-413.

[74] Burhenne L, Damiani M, Aicher T. Effect of feedstock water content and pyrolysis temperature on the structure and reactivity of spruce wood char produced in fixed bed pyrolysis. Fuel, 2013, 107: 836-847.

[75] Fu P, Hu S, Xiang J, Sun L S, Li P S, Zhang J Y, Zheng C G. Pyrolysis of maize stalk on the characterization of chars formed under different devolatilization conditions. Energy Fuel, 2009, 23: 4605-4611.
[76] Chen Y, Zhang X, Chen W, Yang H, Chen H. The structure evolution of biochar from biomass pyrolysis and its correlation with gas pollutant adsorption performance. Bioresour Technol, 2017, 246: 101-109.

[77] Zhang H, Voroney R, Price G. Effects of temperature and processing conditions on biochar chemical properties and their influence on soil C and N transformations. Soil Biol Biochem, 2015, 83: 19-28.

[78] Cao X D, Harris W. Properties of dairy-manure-derived biochar pertinent to its potential use in remediation. Bioresour Technol, 2010, 101: 5222-5228.

[79] 谢祖彬, 刘琦, 许燕萍, 朱春悟. 生物炭研究进展及其研究方 向. 土壤, 2011, 43: 857-861.

Xie Z B, Liu Q, Xu Y P, Zhu C W. Advances and perspectives of biochar research. Soil, 2011, 43: 857-861 (in Chinese with English abstract).

[80] 陈静文, 张迪, 吴敏, 王朋. 两类生物炭的元素组分分析及其 热稳定性. 环境化学, 2014, 33: 417-422.

Chen J W, Zhang D, Wu M, Wang P. Elemental composition and thermal stability of two different biochars. Environ Chem, 2014, 33: 417-422 (in Chinese with English abstract).

[81] Zornoza R, Moreno-Barriga F, Acosta J A, Muñoz M A, Faz A. Stability, nutrient availability and hydrophobicity of biochars derived from manure, crop residues, and municipal solid waste for their use as soil amendments. Chemosphere, 2016, 144: 122-130.

[82] Hossain M, Strezov V, Chan K Y, Ziolkowski A, Nelson P F. Influence of pyrolysis temperature on production and nutrient properties of wastewater sludge biochar. J Environ Manage, 2011, 92: 223-228.

[83] Chen T, Zhang Y X, Wang H T, Lu W J, Zhou Z Y, Zhang Y C, Ren L L. Influence of pyrolysis temperature on characteristics and heavy metal adsorptive performance of biochar derived from municipal sewage sludge. Bioresour Technol, 2014, 164: 47-54.

[84] Subedi R, Taupe N, Pelissetti S, Petruzzelli L, Bertora C, Leahy J, Grignani C. Greenhouse gas emissions and soil properties following amendment with manure-derived biochars: influence of pyrolysis temperature and feedstock type. J Environ Manag, 2016, 166: 73-83.

[85] Zhao L, Cao X D, Masek O, Zimmerman A. Heterogeneity of biochar properties as a function of feedstock sources and production temperatures. J Hazard Mater, 2013, 256/257: 1-9.

[86] Ahmad M, Lee S S, Dou X, Mohan D, Sung J K, Yang J E, Ok Y S. Effects of pyrolysis temperature on soybean stover- and peanut shell-derived biochar properties and TCE adsorption in water. Bioresour Technol, 2012, 118: 536-544.

[87] Tan X F, Liu Y G, Zeng G M, Wang X, Hu X J, Gu Y L, Yang Z Z. Application of biochar for the removal of pollutants from aqueous solutions. Chemosphere, 2015, 125: 70-85.

[88] Zhang J, Wang Q. Sustainable mechanisms of biochar derived from brewers' spent grain and sewage sludge for ammonia-nitrogen capture. J Clean Prod, 2016, 112: 3927-3934.

[89] 张旭东, 梁超, 诸葛玉平, 姜勇, 解宏图, 何红波, 王晶. 黑碳 在土壤有机碳生物地球化学循环中的作用. 土壤通报, 2003, 34: 349-355.

Zhang X D, Liang, Zhu-Ge Y P, Jiang Y, Jie H T, He H B, Wang J. Roles of black carbon in the biogeochemical cycles of soil 
organic carbon. Chin J Soil Sci, 2003, 34: 349-355 (in Chinese with English abstract).

[90] Ameloot N, Graber E R, Verheijen F G A, Neve S D. Interactions between biochar stability and soil organisms: review and research needs. Eur J Soil Sci, 2013, 64: 379-390.

[91] Huff M D, Kumar S, Lee J W. Comparative analysis of pinewood, peanut shell, and bamboo biomass derived biochars produced via hydrothermal conversion and pyrolysis. J Environ Manage, 2014, 146: 303-308.

[92] Gul S, Whalen J K, Thomas B W, Sachdeva V, Deng H. Physico-chemical properties and microbial responses in biochar-amended soils: mechanisms and future directions. Agric Ecosyst Environ, 2015, 206: 46-59.

[93] Gul S, Whalen J K. Biochemical cycling of nitrogen and phosphorus in biochar-amended Soils. Soil Biol Biochem, 2016, 103: $1-15$.

[94] Spokas K A, Novak J M, Venterea R T. Biochar's role as an alternative N fertilizer: ammonia capture. Plant Soil, 2012, 350: $35-42$.

[95] Mukherjee A, Zimmerman A R. Organic carbon and nutrient release from a range of laboratory-produced biochars and biochar-soil mixtures. Geoderma, 2013, 193: 122-130.

[96] Zheng H, Wang Z Y, Deng X, Zhao J, Luo Y, Novak J, Herbert S, Xing B S. Characteristics and nutrient values of biochars produced from giant reed at different temperatures. Bioresour Technol, 2013, 130: 463-471.

[97] Ding Y, Liu Y G, Liu S B, Li Z W, Tan X F, Huang X X, Zeng G M, Zhou L, Zheng B H. Biochar to improve soil fertility. A review. Agron Sustain Dev, 2016, 36: 36.

[98] Silber A, Levkovitch I, Graber E R. PH-dependent mineral release and surface properties of cornstraw biochar: agronomic implications. Environ Sci Technol, 2010, 44: 9318-9323.

[99] Cao X D, Ma L Q, Gao B, Harris W. Dairy-manure derived biochar effectively sorbs lead and atrazine. Environ Sci Technol, 2009, 43: 3285-3291.

[100] Yuan J H, Xu R K, Zhang H. The forms of alkalis in the biochar produced from crop residues at different temperatures. Bioresour Technol, 2011, 102: 3488-3497.

[101] Wang Z Y, Liu G C, Zheng H, Li F M, Ngo H H, Guo W S, Liu C, Chen L, Xing B S. Investigating the mechanisms of biochar's removal of lead from solution. Bioresour Technol, 2015 177: 308-317.

[102] Hussain M, Farooq M, Nawaz A, Al-Sadi A M, Solaiman Z M, Alghamdi S S, Ammara U, Ok Y S, Siddique K H M. Biochar for crop production: potential benefits and risks. $J$ Soil Sediment, 2017, 17: 685-716.

[103] Lyu H, He Y, Tang J, Hecker M, Liu Q, Jones P D, Codling G, Giesy J P. Effect of pyrolysis temperature on potential toxicity of biochar if applied to the environment. Environ Pollut, 2016, 218: $1-7$.

[104] Kookana R S, Sarmah A K, Van Zwieten L, Krull E, Singh B. Biochar application to soil: agronomic and environmental benefits and unintended consequences. Adv Agron, 2011, 112: 103-143.

[105] McGrath T E, Wooten J B, Chan W G, Hajaligol M R. Formation of polycyclic aromatic hydrocarbons from tobacco: the link between low temperature residual solid (char) and PAH formation. Food Chem Toxicol, 2007, 45: 1039-1050.

[106] Buss W, Graham M, MacKinnon G, Mašek O. Strategies for producing biochars with minimum PAH contamination. $J$ Anal Appl Pyrol, 2016, 119: 24-30.

[107] Wang C, Wang Y, Herath H. Polycyclic aromatic hydrocarbons (PAHs) in biochar - their formation, occurrence and analysis: a review. Org Geochem, 2017, 114: 1-11.

[108] Brown R A, Kercher A K, Nguyen T H, Nagle D C, Ball W P. Production and characterization of synthetic wood chars for use as surrogates for natural sorbents. Org Geochem, 2006, 37: 321-333.

[109] Huang H, Yao W, Lia R, Ali A, Du J, Guo D, Xiao R, Guo Z, Zhang Z, Awasthi M K. Effect of pyrolysis temperature on chemical form, behavior and environmental risk of $\mathrm{Zn}, \mathrm{Pb}$ and $\mathrm{Cd}$ in biochar produced from phytoremediation residue. Bioresour Technol, 2018, 249: 487-493.

[110] Wang X, Li C, Li Z, Yu G, Wang Y. Effect of pyrolysis temperature on characteristics, chemical speciation and risk evaluation of heavy metals in biochar derived from textile dyeing sludge. Ecotox Environ Safe, 2019, 168: 45-52.

[111] Standardized Product Definition and Product Testing Guidelines for Biochar That is Used in Soil (aka IBI Biochar Standards) Version 2.1, 2015. pp 14-15.

[112] Liu Z, Wang L, Xiao H, Guo X, Urbanovich O, Nagorskaya L, Li X. A review on control factors of pyrolysis technology for plants containing heavy metals. Ecotox Environ Safe, 2020, 191: 110181.

[113] 陈温福, 张伟明, 孟军. 农用生物炭研究进展与前景. 中国 农业科学, 2013, 46: 3324-3333.

Chen W F, Zhang W M, Meng J. Advances and prospects in research of biochar utilization in agriculture. Sci Agric Sin, 2013, 46: 3324-3333 (in Chinese with English abstract).

[114] Xu X Y, Cao X D, Zhao L, Wang H L, Yu H R, Gao B. Removal of $\mathrm{Cu}, \mathrm{Zn}$, and $\mathrm{Cd}$ from aqueous solutions by the dairy manure-derived biochar. Environ Sci Pollut $R$, 2013, 20: 358-368.

[115] Zhu L, Lei H W, Wang L, Yadavalli G, Zhang X S, Wei Y, Liu Y P, Yan D, Chen S L, Ahring B. Biochar of corn stover: microwave-assisted pyrolysis condition induced changes in surface functional groups and characteristics. J Anal Appl Pyrol, 2015, 115: 149-156.

[116] Antonherrero R, Garciadelgado C, Alonsoizquierdo M, Garciarodriguez G, Cuevas J, Eymar E. Comparative adsorption of tetracyclines on biochars and stevensite: looking for the most effective adsorbent. Appl Clay Sci, 2018, 160: 162-172.

[117] Koutcheiko S, Monreal C M, Kodama H, McCracken T, Kotlyar L. Preparation and characterization of activated carbon derived from the thermo-chemical conversion of chicken manure. Bioresour Technol, 2007, 98: 2459-2464.

[118] Fu P, Hu S, Xiang J, Sun L S, Su S, An S M. Study on the gas evolution and char structural change during pyrolysis of cotton stalk. J Anal Appl Pyrol, 2012, 97: 130-136.

[119] Weber K, Quicker P. Properties of biochar. Fuel, 2018, 217 : 240-261.

[120] Liang B, Lehmann J, Solomon D, Kinyangi J, Grossman J M, 
Oneill B, Skjemstad J O, Thies J E, Luizao F J, Petersen J B, Neves E G. Black carbon increases cation exchange capacity in soils. Soil Sci Soc Am J, 2006, 70: 1719-1730.

[121] Lee J W, Kidder M, Evans B R. Characterization of biochars produced from corn stovers for soil amendment. Environ Sci Technol, 2010, 44: 7970-7974.

[122] Inyang M, Gao B, Pullammanappallil P, Ding W, Zimmerman A R. Biochar from anaerobically digested sugarcane bagasse. Bioresour Technol, 2010, 101: 8868-8872.

[123] Suliman W, Harsh J B, Fortuna A, Garciaperez M, Abulail N I. Quantitative effects of biochar oxidation and pyrolysis temperature on the transport of pathogenic and nonpathogenic Escherichia coli in biochar-amended sand columns. Environ Sci Technol, 2017, 51: 5071-5081.

[124] Mukherjee A, Zimmerman A R, Harris W. Surface chemistry variations among a series of laboratory-produced biochars. Geoderma, 2011, 163: 247-255.

[125] Yu K L, Lau B F, Show P L, Ong H C, Ling T C, Chen W, Ng E $\mathrm{P}$, Chang J. Recent developments on algal biochar production and characterization. Bioresour Technol, 2017, 246: 2-11.

[126] Li L C, Zou D S, Xiao Z H, Zeng X Y, Zhang L Q, Jiang L D, Wang A D, Ge D B, Zhang G L, Liu F. Biochar as a sorbent for emerging contaminants enables improvements in waste management and sustainable resource use. J Clean Prod, 2019, 210: 1324-1342.

[127] Lian F, Xing B S. Black carbon (biochar) in water/soil environments: molecular structure, sorption, stability, and potential risk. Environ Sci Technol, 2017, 51: 13517-13532.

[128] Wang H Y, Gao B, Fang J, Ok Y S, Xue Y W, Yang K, Cao X D. Engineered biochar derived from eggshell-treated biomass for removal of aqueous lead. Ecol Eng, 2018, 121: 124-129.

[129] Khare P, Dilshad U, Rout P K, Yadav V, Jain S. Plant refuses driven biochar: application as metal adsorbent from acidic solutions. Arab J Chem, 2013, 10(S2): S3054-S3063.

[130] 陈温福, 张伟明, 孟军. 生物炭与农业环境研究回顾与展望. 农业环境科学学报, 2014, 33: 821-828.

Chen W F, Zhang W M, Meng J. Biochar and agro-ecological environment: review and prospect. J Agro-Environ Sci, 2014, 33 : 821-828 (in Chinese with English abstract).

[131] Chen B L, Zhou D D, Zhu L Z. Transitional adsorption and partition of nonpolar and polar aromatic contaminants by biochars of pine needles with different pyrolytic temperatures. Environ Sci Technol, 2008, 42: 5137-5143.

[132] El-Naggar A, Lee S S, Rinklebe J, Farooq M, Song H, Sarmah A K, Zimmerman A R, Ahmad M, Shaheen S M, Ok Y S. Biochar application to low fertility soils: a review of current status, and future prospects. Geoderma, 2019, 337: 836-554.

[133] Bruun E W. Application of fast pyrolysis biochar to a loamy soil-effects on carbon and nitrogen dynamics and potential for carbon sequestration. PhD thesis. Technical Univ. of Denmark, 2800 Kgs. Lyngby, 2011.

[134] Gray M, Johnson M G, Dragila M I, Kleber M. Water uptake in biochars: the roles of porosity and hydrophobicity. Biomass Bioenerg, 2014, 61: 196-205.

[135] Fang Q, Chen B, Lin Y, Guan Y. Aromatic and hydrophobic surfaces of wood-derived biochar enhance perchlorate adsorp- tion via hydrogen bonding to oxygen-containing organic groups. Environ Sci Technol, 2014, 48: 279-288.

[136] Das O, Sarmah A K. The love-hate relationship of pyrolysis biochar and water: a perspective. Sci Total Environ, 2015, 512/513: 682-685.

[137] Zhang J, You C. Water holding capacity and absorption properties of wood chars. Energ Fuel, 2013, 27: 2643-2648.

[138] Wiedemeier D B, Abiven S, Hockaday W C, Keiluweit M, Kleber M, Masiello C A, McBeath A V, Nico P S, Pyle L A, Schneider M P W, Smernik R J, Wiesenberg G L B, Schmidt M W I. Aromaticity and degree of aromatic condensation of char. Org Geochem, 2015, 78: 135-143.

[139] Manyà J J, Ortigosa M A, Laguarta S, Manso J A. Experimental study on the effect of pyrolysis pressure, peak temperature, and particle size on the potential stability of vine shoots-derived biochar. Fuel, 2014, 133: 163-172.

[140] Kuhlbusch T A J. Method for determining black carbon in vegetation fire residues. Environ Sci Technol, 1995, 29: 2695-2702.

[141] Spokas K. Review of the stability of biochar in soils: predictability of O:C molar ratios. Carbon Manage, 2010, 1: 289-303.

[142] Li W, Dang Q, Brown R C, Laird D, Wright M M. The impacts of biomass properties on pyrolysis yields, economic and environmental performance of the pyrolysis-bioenergy-biochar platform to carbon negative energy. Bioresour Technol, 2017, 241: 959-968.

[143] Singh B P, Cowie A L, Smernik R J. Biochar carbon stability in a clayey soil as a function of feedstock and pyrolysis temperature. Environ Sci Technol, 2012, 46: 11770-11778.

[144] Han L F, Ro K S, Wang Y, Sun K, Sun H R, Libra J A, Xing B S. Oxidation resistance of biochars as a function of feedstock and pyrolysis condition. Sci Total Environ, 2018, 616/617: 335-344.

[145] Chen J, Li S, Liang C, Xu Q, Li Y, Qin H, Fuhrmann J J. Response of microbial community structure and function to short-term biochar amendment in an intensively managed bamboo (Phyllostachys praecox) plantation soil: effect of particle size and addition rate. Sci Total Environ, 2017, 574: 24-33.

[146] Sigua G C, Novak J M, Watts D W, Cantrell K B, Shumaker P D, Szögi A A, Johnson M G. Carbon mineralization in two ultisols amended with different sources and particle sizes of pyrolyzed biochar. Chemosphere, 2014, 103: 313-321.

[147] Crombie K, Mašek O. Pyrolysis biochar systems, balance between bioenergy and carbon sequestration. GCB Bioenergy, 2015, 7: 349-361.

[148] Wang B, Gao B, Fang J. Recent advances in engineered biochar productions and applications. Crit Rev Env Sci Tec, 2017, 47: 2158-2207.

[149] Wang T, Liu X Q, Men Q Y, Ma C C, Liu Y, Ma W, Liu Z, Wei M B, Li C X, Yan Y S. Surface plasmon resonance effect of Ag nanoparticles for improving the photocatalytic performance of biochar quantum-dot $/ \mathrm{Bi}_{4} \mathrm{Ti}_{3} \mathrm{O}_{12}$ nanosheets. Chin J Catal, 2019, 40: 886-894.

[150] 左卫元, 全海娟, 史兵方, 陈盛余, 段艳, 廖安平. 生物炭/锰 氧化物复合材料对苯甲酸的吸附研究. 无机盐工业, 2018, 50(8): 57-61.

Zhuo W Y, Tong H G, Shi B F, Chen S Y, Duan Y, Liao A P. Adsorption effect of biochar/manganese oxide composite mate- 
rial on benzoic acid. Inorg Chem Ind, 2018, 50(8): 57-61 (in Chinese with English abstract).

[151] Hu X, Ding Z, Zimmerman A R, Wang S, Gao B. Batch and column sorption of arsenic onto iron-impregnated biochar synthesized through hydrolysis. Water Res, 2015, 68: 206-216.

[152] Thines K R, Abdullah E C, Mubarak N M, Ruthiraan M. Synthesis of magnetic biochar from agricultural waste biomass to enhancing route for waste water and polymer application. Renew Sust Energ Rev, 2017 67: 257-276.

[153] Zhang M, Gao B, Varnoosfaderani S, Hebard A, Yao Y, Inyang M. Preparation and characterization of a novel magnetic biochar for arsenic removal. Bioresour Technol, 2013, 130: 457-462.

[154] 吕宏虹, 宫艳艳, 唐景春, 黄耀, 高凯. 生物炭及其复合材料 的制备与应用研究进展. 农业环境科学学报, 2015, 34: 1429-1440.

Lyu H H, Gong Y Y, Tang J C, Huang Y, Gao K. Advances in preparation and applications of biochar and its composites. $J$ Agro-Environ Sci, 2015, 34: 1429-1440 (in Chinese with English abstract).

[155] Chen B, Chen Z, Lyu S. A novel magnetic biochar efficiently sorbs organic pollutants and phosphate. Bioresour Technol, 2011, 102: 716-723.

[156] Trakal L, Veselsk V, Safak I, Vtkov M, Chalov S, Komarek M. Lead and cadmium sorption mechanisms on magnetically modified biochars. Bioresour Technol, 2016, 203: 318-324.

[157] Uchimiya M, Bannon D I, Wartelle L H. Retention of heavy metals by carboxyl functional groups of biochars in small arms range soil. J Agric Food Chem, 2012, 60: 1798-1809.

[158] Huff M D, Lee J W. Biochar-surface oxygenation with hydrogen peroxide. J Environ Manage, 2016, 165: 17-21.

[159] Xue Y, Gao B, Yao Y, Inyang M, Zhang M, Zimmerman A R, Ro K S. Hydrogen peroxide modification enhances the ability of biochar (hydrochar) produced from hydrothermal carbonization of peanut hull to remove aqueous heavy metals: batch and column tests. Chem Eng J, 2012, 200-202: 673-680.

[160] Vithanage M, Rajapaksha A U, Zhang M, Thiele-Bruhn S, Lee S S, Ok Y S. Acid-activated biochar increased sulfamethazine retention in soils. Environ Sci Pollut R, 2015, 22: 2175-2186.

[161] Hadjittofi L, Prodromou M, Pashalidis I. Activated biochar derived from cactus fibres: preparation, characterization and application on $\mathrm{Cu}(\mathrm{II})$ removal from aqueous solutions. Bioresour Technol, 2014, 159: 460-464.

[162] Ding Z, Hu X, Wan Y, Wang S, Gao B. Removal of lead, copper, cadmium, zinc, and nickel from aqueous solutions by alkalimodified biochar: Batch and column tests. J Ind Eng Chem, 2016, 33: 239-245

[163] Fan Y, Wang B, Yuan S, Wu X, Chen J, Wang L. Adsorptive removal of chloramphenicol from wastewater by $\mathrm{NaOH}$ modified bamboo charcoal. Bioresour Technol, 2010, 101: 7661-7664.

[164] Li B, Yang L, Wang C Q, Zhang Q P, Liu Q C, Li Y D, Xiao R. Adsorption of Cd (II) from aqueous solutions by rape straw biochar derived from different modification processes. Chemosphere, 2017, 175: 332-340.

[165] Dehkhoda A M, Ellis N, Gyenge E. Effect of activated biochar porous structure on the capacitive deionization of $\mathrm{NaCl}$ and $\mathrm{ZnCl}_{2}$ solutions. Micropor Mesopor Mat, 2016, 224: 217-228.
[166] Regmi P, Moscoso J L G, Kumar S, Cao X Y, Mao J D, Schafran G. Removal of copper and cadmium from aqueous solution using switchgrass biochar produced via hydrothermal carbonization process. J Environ Manage, 2012, 109: 61-69.

[167] Ahmed M B, Zhou J L, Ngo H H, Guo W S, Chen M F. Progress in the preparation and application of modified biochar for improved contaminant removal from water and wastewater. Bioresour Technol, 2016, 214: 836-851.

[168] Samsuri A W, Sadegh-Zadeh F, She-Bardan B J. Adsorption of $\mathrm{As}(\mathrm{III})$ and $\mathrm{As}(\mathrm{V})$ by $\mathrm{Fe}$ coated biochars and biochars produced from empty fruit bunch and rice husk. J Environ Chem Eng, 2013, 1: 981-988.

[169] Wang Y, Wang X J, Liu M, Wang X, Wu Z, Yang L Z, Xia S Q, Zhao J F. Cr(VI) removal from water using cobalt-coated bamboo charcoal prepared with microwave heating. Ind Crops Prod, 2012, 39: 81-88.

[170] Zhang M, Gao B, Yao Y, Inyang M. Phosphate removal ability of biochar/MgAl-LDHultra-fine composites prepared by liquid-phase deposition. Chemosphere, 2013, 92: 1042-1047.

[171] Ma Y, Liu W J, Zhang N, Li Y S, Jiang H, Sheng G P. Polyethylenimine modified biochar adsorbent for hexavalent chromium removal from the aqueous solution. Bioresour Techn, 2014, 169: 403-408.

[172] Divband Hafshejani L, Hooshmand A, Naseri A A, Mohammadia A S, Abbasib F, Bhatnagarc A. Removal of nitrate from aqueous solution by modified sugarcane bagasse biochar. Ecolog Engin, 2016, 95: 101-111.

[173] Zhu S S, Huang X C, Wang D W, Wang L, Ma F. Enhanced hexavalent chromium removal performance and stabilization by magnetic iron nanoparticles assisted biochar in aqueous solution: mechanisms and application potential. Chemosphere, 2018, 207: $50-59$.

[174] 朱丹丹, 周启星. 功能纳米材料在重金属污染水体修复中的 应用研究进展. 农业环境科学学报, 2018, 37: 1551-1564.

Zhu D D, Zhou Q X. A review on the removal of heavy metals from water using nanomaterials. J Agro-Environ Sci, 2018, 37: 1551-1564 (in Chinese with English abstract).

[175] Tan X F, Liu Y G, Gu Y L, Xu Y, Zeng G M, Hu X J, Liu S B, Wang X, Liu S M, Li J. Biochar-based nano-composites for the decontamination of wastewater: a review. Bioresour Technol, 2016, 212: 318-333

[176] 蒲生彦, 贺玲玲, 刘世宾. 生物炭复合材料在废水处理中的 应用研究进展. 工业水处理, 2019, 39(9): 1-8.

$\mathrm{Pu} \mathrm{S} \mathrm{Y,} \mathrm{He} \mathrm{L} \mathrm{L,} \mathrm{Liu} \mathrm{S} \mathrm{B.} \mathrm{Review} \mathrm{on} \mathrm{the} \mathrm{preparation} \mathrm{of} \mathrm{biochar}$ composites and its applications in wastewater treatment. Ind Water Treat, 2019, 39(9): 1-8 (in Chinese with English abstract).

[177] Rajapaksha A U, Chen S S, Tsang D C W, Zhang M, Vithanage M, Mandal S, Gao B, Bolan N S, Ok Y S. Engineered/designer biochar for contaminant removal/immobilization from soil and water: potential and implication of biochar modification Chemosphere, 2016, 148: 276-291.

[178] Rajapaksha A U, Vithanage M, Ahmad M, Seo D C, Cho J S, Lee S E, Lee S S, Ok Y S. Enhanced sulfamethazine removal by steam-activated invasive plant derived biochar. J Hazard Mater, 2015, 290: 43-50.

[179] Zhang C S, Liu L, Zhao M H, Rong H W, Xu Y. The environ- 
mental characteristics and applications of biochar. Environ Sci Pollut R, 2018, 25: 21525-21534.

[180] Fungo B, Guerena D, Thiongo M, Lehmann J, Neufeldt H, Kalbitz $\mathrm{K} . \mathrm{N}_{2} \mathrm{O}$ and $\mathrm{CH}_{4}$ emission from soil amended with steam-activated biochar. J Plant Nutr Soil Sc, 2014, 177: 34-38.

[181] Lima I M, Marshall W E. Adsorption of selected environmentally important metals by poultry manure-based granular activated carbons. Chem Technol Biot, 2005, 80: 1054-1061.

[182] De M, Azargohar R, Dalai A K, Shewchuk S R. Mercury removal by bio-char based modified activated carbons. Fuel, 2013, 103: 570-578.

[183] Borchard N, Wolf A, Laabs V, Aeckersberg R, Scherer H, Moeller A, Amelung W. Physical activation of biochar and its meaning for soil fertility and nutrient leaching: a greenhouse experiment. Soil Use Manage, 2012, 28: 177-184.

[184] Foo K Y, Hameed B H. Preparation and characterization of activated carbon from pistachio nut shells via microwave-induced chemical activation. Biomass Bioenergy, 2011, 35: 3257-3261.

[185] 焚兴君, 尤进茂, 谭干祖, 俞贤达, 焦天权. 微波促进有机化 学反应研究进展. 化学进展, 1998, (3): 51-61.

Fan X J, You J M, Tan G Z, Yu X D, Jiao T Q. Progress in microwave-organic reaction enhancement chemistry. Prog Chem, 1998, (3): 51-61.

[186] Wan Y, Chen P, Zhang B, Yang C, Liu Y, Lin X, Ruan R. Microwave-assisted pyrolysis of biomass: catalysts to improve product selectivity. J Anal Appl Pyrol, 2009, 86: 161-167.

[187] Mohamed B A, Ellis N, Kim C S, Bi X, Emam A E R. Engineered biochar from microwave-assisted catalytic pyrolysis of switchgrass for increasing water holding capacity and fertility of sandy soil. Sci Total Environ, 2016, 566/567: 387-397.

[188] Du Z, Zheng T, Wang P, Hao L, Wang Y. Fast microwave-assisted preparation of a low-cost and recyclable carboxyl modified lignocellulose-biomass jute fiber for enhanced heavy metal removal from water. Bioresour Technol, 2016, 201: 41-49.

[189] Shen B, Li G, Wang F, Wang Y, He C, Zhang M, Singh S. Elemental mercury removal by the modified bio-char from medicinal residues. Chem Eng J, 2015, 272: 28-37.

[190] Li G, Shen B, Li F, Tian L, Singh S, Wang F. Elemental mercury removal using biochar pyrolyzed from municipal solid waste. Fuel Process Technol, 2015, 133: 43-50.
[191] Menendez J A, Inguanzo M, Pis J J. Microwave-induced pyrolysis of sewage sludge. Water Res, 2002, 36: 3261-3264.

[192] Lyu H, Gao B, He F, Ding C, Tang J, Crittenden J C. Ball-milled carbon nanomaterials for energy and environmental applications. ACS Sustain Chem Eng, 2017, 5: 9568-9585.

[193] Shan D, Deng S, Zhao T, Wang B, Wang Y, Huang J, Yu G, Winglee J, Wiesner M R. Preparation of ultrafine magnetic biochar and activated carbon for pharmaceutical adsorption and subsequent degradation by ball milling. J Hazard Mater, 2016, 305: 156-163.

[194] Cai H, Xu L, Chen G, Peng C, Ke F, Liu Z, Li D, Zhang Z, Wan $X$. Removal of fluoride from drinking water using modified ultrafine tea powder processed using a ball mill. Appl Surf Sci, 2016, 375: 74-84.

[195] Lyu H, Gao B, He F, Zimmerman A, Ding C, Huang H, Tang J. Effects of ball milling on the physicochemical and sorptive properties of biochar: experimental observations and governing mechanisms. Environ Poll, 2018, 233: 54-63.

[196] Peterson S C, Jackson M A, Kim S, Palmquist D E. Increasing biochar surface area: optimization of ball milling parameters. Powder Technol, 2012, 228: 115-120.

[197] Wang D, Zhang W, Hao X, Zhou D. Transport of biochar particles in saturated granular media: effects of pyrolysis temperature and particle size. Environ Sci Technol, 2013, 47: 821-828.

[198] Chen M, Wang D, Yang F, Xu X, Xu N, Cao X. Transport and retention of biochar nanoparticles in a paddy soil under environmentally-relevant solution chemistry conditions. Environ Pollut, 2017, 230: 540-549.

[199] 陈健康. 紫外辐射改性碳材料对水中重金属的吸附研究. 重 庆大学硕士学位论文, 重庆, 2014.

Chen J K. The Study of Adsorption Heavy Metals from Aqueous Solution Using Ultraviolet Radiation Modified Carbon Materials. MS Thesis of Chongqing University, Chongqing, China, 2014 (in Chinese with English abstract).

[200] 李桥, 高屿涛, 姜蔚, 雍毅. 紫外辐照改性生物炭对土壤中 Cd 的稳定化效果. 环境工程学报, 2017, 11: 5708-5714.

Li Q, Gao Y T, Jiang W, Yong Y. Stabilization of Cd contaminated soil by ultraviolet irradiation modified biochar. Chin J Environ Engin, 2017, 11: 5708-5714 (in Chinese with English abstract). 